PHYSICAL REVIEW D 96, 123508 (2017)

\title{
Novel approach toward the large-scale stable interacting dark-energy models and their astronomical bounds
}

\author{
Weiqiang Yang, ${ }^{1, *}$ Supriya Pan, ${ }^{2,3, \dagger}$ and David F. Mota ${ }^{4, *}$ \\ ${ }^{1}$ Department of Physics, Liaoning Normal University, Dalian 116029, People's Republic of China \\ ${ }^{2}$ Department of Physical Sciences, Indian Institute of Science Education and Research, \\ Kolkata, Mohanpur 741246, West Bengal, India \\ ${ }^{3}$ Department of Mathematics, Raiganj Surendranath Mahavidyalaya, \\ Sudarshanpur, Raiganj, West Bengal 733134, India \\ ${ }^{4}$ Institute of Theoretical Astrophysics, University of Oslo, 0315 Oslo, Norway
}

(Received 4 September 2017; published 5 December 2017)

\begin{abstract}
Stability analysis of interacting dark-energy models generally divides its parameters space into two regions, (i) $w_{x} \geq-1$ and $\xi \geq 0$ and (ii) $w_{x} \leq-1$ and $\xi \leq 0$, where $w_{x}$ is the dark-energy equation of state and $\xi$ is the coupling strength of the interaction. Because of this separation, crucial information about the cosmology and phenomenology of these models may be lost. In a recent study, it has been shown that one can unify the two regions with a coupling function that depends on the dark-energy equation of state. In this work, we introduce a new coupling function that also unifies the two regions of the parameter space and generalizes the previous proposal. We analyze this scenario considering the equation of state of dark energy to be either constant or dynamical. We study the cosmology of such models and constrain both scenarios with the use of the latest astronomical data from both the background evolution as well as large-scale structures. Our analysis shows that a nonzero value of the coupling parameter $\xi$ as well as the dark-energy equation of state other than -1 are allowed. However, within $1 \sigma$ confidence level, $\xi=0$ and the darkenergy equation of state equal to -1 are compatible with the current data. In other words, the observational data allow a very small but nonzero deviation from the $\Lambda$ cosmology; however, within the $1 \sigma$ confidence region, the interacting models can mimic the $\Lambda$ cosmology. In fact, we observe that the models both at the background and perturbative levels are very hard to distinguish from each other and from $\Lambda$ cosmology as well. Finally, we offer a rigorous analysis on the current tension on $H_{0}$, allowing different regions of the dark-energy equation of state, which shows that interacting dark-energy models reasonably solve the current tension on $H_{0}$.
\end{abstract}

DOI: $10.1103 /$ PhysRevD.96.123508

\section{INTRODUCTION}

Dark matter (DM) and dark energy (DE) are the two heavy constituents of our Universe [1]. The first one, i.e., the dark matter, is believed to be responsible for the structure formation of the universe and is almost pressureless, while dark energy, a modification of the matter sector in the general relativity, is assumed to be responsible for the current observed acceleration of the universe. Both of them comprise approximately $96 \%$ of the total energy density of the universe with unknown character and origin. Thus, the dynamics of the universe heavily rests on this sector. Now, since the character of this dark sector is unknown, thus, sometimes it is assumed that perhaps the dark matter and dark energy are coupled to each other so that they behave like a single dark fluid. Although this consideration sounds slightly phenomenological, but such a possibility cannot be ruled out in any ways. From the philosophical point of

\footnotetext{
d11102004@163.com

span@iiserkol.ac.in

mota@astro.uio.no
}

view, we do not have any strong evidence to exclude the phenomenon of dark matter-dark energy interaction. So, one can of course think of some interaction between these two fields. In fact, the standard cosmological laws can be retrieved at any time under the no-interaction limit. Additionally, the dynamics of the universe in the presence of any coupling between dark matter and dark energy becomes quite richer with many possibilities. On the other hand, from the theory of particle physics, any two fields can interact with each other. Since both dark energy and dark matter can be thought to be of some fields, for instance, some scalar field, the idea behind the dark matter-dark energy interaction is also supported from the particle physics view point. This henceforth initiated a new branch in the physics of dark energy in the name of interacting dark-energy theory. The idea of coupling in the dark sectors was initiated by Wetterich [2] and subsequently by Amendola [3]. Thus, so far, this particular scenario has been explored in the context of current cosmology with some interesting outcomes. The coupling between the dark matter and the dark energy may provide an explanation to the cosmic coincidence problem [4], a generic problem in 
the dynamical dark-energy models and even in the $\Lambda$-Cold Dark Matter $(\Lambda \mathrm{CDM})$ cosmology. Thus, in the last couple of years, a rigorous analysis has been performed by several authors with many interesting possibilities; see, for instance, Refs. [5-38]. The investigations in coupled dark energy were further fueled when the latest observational data estimated a nonzero interaction in the dark sector [39-44]. Although most of the studies in this direction automatically assume the interaction between dark matter and dark energy, a generalized version of the interacting dark energy, in which the interacting components could be any two barotropic fluids, is also appealing [45].

In this work, we propose a novel mechanism to test the stability of interacting dark-energy models in which the dark-energy equation of state can be unrestricted, unlike other interacting dark-energy models in which the state parameter for dark energy is necessarily restricted. To illustrate more in this direction, we consider a spatially flat Friedmann-Lemaître-Robertson-Walker universe in which pressureless dark matter interacts with dark energy through a nongravitational interaction function. We find that, using the pressure perturbation equation for dark energy, it is quite possible to construct several interaction models that can be tested without any prior limitation imposed on the dark-energy equation of state. The newly constructed interaction functions do have a direct dependence on the dark-energy equation of state. However, the entire picture becomes exciting when the interaction function automatically contains such dependence on the dark-energy equation of state. We examine the interacting scenario both for the constant and dynamical nature of the dark-energy equation of state. Finally, we constrain both the interacting scenarios using the latest observational data from different astronomical sources, namely, the cosmic microwave background radiation [46,47], baryon acoustic oscillations distance measurements [48-50], joint light curve analysis from Supernovae Type Ia [51], weak lensing [52,53], redshift space distortion data [54-59], and finally the Hubble parameter measurements from cosmic chronometers [60] plus the local Hubble constant [61]. We note that the use of several observational data provides better and tight constraints on the models.

The paper is organized as follows. In Sec. II, we describe the background equations of an interacting universe with the new interacting dark-energy model with constant and dynamical dark-energy equation of state. Thus, essentially, we focus on two interacting cosmological scenarios in this study. In Sec. III, we discuss the interacting cosmology in the perturbative universe. In Sec. IV, we describe the astronomical data sets that have been used to constrain the models, and Sec. V follows the observational constraints of the proposed models. After that, in Sec. VI, we compare the current models from the statistical ground and also make a comparison with other existing interaction models. Section VII contains an extensive analysis on the current tension on $H_{0}$. Finally, we close the work in Sec. VIII with a short summary of the results obtained.

\section{INTERACTING COSMOLOGY: BACKGROUND UNIVERSE}

In this section, we describe the governing equations for any interacting dark-energy model. The background geometry is set to be a spatially flat Friedmann-LemaîtreRobertson-Walker (FLRW) universe characterized by the line element $\quad d s^{2}=-d t^{2}+a^{2}(t)\left[d r^{2}+r^{2}\left(d \theta^{2}+\sin ^{2} \theta d \phi^{2}\right)\right]$, where $a(t)$ is the expansion scale factor of the universe. The conservation equation of a coupled dark-matter and dark-energy system in the FLRW universe can be written as, $\dot{\rho}_{c}+3 H \rho_{c}=-\dot{\rho}_{x}-3 H\left(p_{x}+\rho_{x}\right)$, which can be decoupled into

$$
\begin{aligned}
\rho_{c}^{\prime}+3 \mathcal{H} \rho_{c} & =-a Q, \\
\rho_{x}^{\prime}+3 \mathcal{H}\left(p_{x}+\rho_{x}\right) & =a Q,
\end{aligned}
$$

with a new quantity $Q$, known as the interaction rate between the dark sectors. Here, $\mathcal{H}=a^{\prime} / a$ is the conformal Hubble parameter in which the prime denotes the differentiation with respect to the conformal time; $\rho_{c}$ and $\rho_{x}$ are, respectively, the energy densities of pressureless dark matter and dark energy; and $p_{x}$ is the pressure of the dark-energy fluid. In addition, we consider the nonrelativistic baryons (energy density $\equiv \rho_{b}$ ) and relativistic radiation (energy density $\equiv \rho_{r}$ ). Since the physics of baryons and radiation are quite well known, we assume that they are conserved independently, which means the evolution laws of baryons and radiation are $\rho_{b} \propto a^{-3}$ and $\rho_{r} \propto a^{-4}$, respectively. The dynamics of the universe is constrained by the Friedmann equation

$$
\left(\frac{\mathcal{H}}{a}\right)^{2}=\left(\frac{8 \pi G}{3}\right)\left(\rho_{b}+\rho_{r}+\rho_{c}+\rho_{x}\right),
$$

which is the constraint equation of the cosmological scenario. Now, to go ahead, one needs a specific functional form for $Q$, and we follow the same tradition. In the literature, several forms of $Q$ exist, and the most used interactions are $Q \propto \rho_{c}$, $Q \propto \rho_{x}, Q \propto\left(\rho_{c}+\rho_{x}\right)$. In this work, we propose a new interaction of the form

$$
Q=\xi \dot{\rho}_{x}=(\xi / a) \rho_{x}^{\prime},
$$

where the dot represents the derivative with respect to the cosmic time, while the prime, as mentioned, stands for the derivative with respect to the conformal time and $\xi$ is the coupling parameter. The direction of energy flow that is determined by the sign of $Q$ is dependent on the sign of the coupling parameter $\xi$ as well as with the evolution of $\rho_{x}$. Precisely, if $\xi>0$, then $Q>0$ whenever $\rho_{x}^{\prime}>0$, and on the other hand, for $\xi>0$, one may obtain $Q<0$ for $\rho_{x}^{\prime}<0$. Similarly, for $\xi<0$, one can also derive the direction of energy flow between the dark sectors from the character of $\rho_{x}^{\prime}$. 
Now, using the conservation equation (2), the interaction (4) can be written in a general form as

$$
Q=\frac{3}{a}\left(\frac{\xi}{\xi-1}\right) \mathcal{H}\left(p_{x}+\rho_{x}\right)=\frac{3 \xi_{e}}{a} \mathcal{H}\left(p_{x}+\rho_{x}\right),
$$

where we call $\xi_{e}=\xi /(1-\xi)$ the effective coupling parameter in the interaction scenario. It is clearly seen from Eq. (5) that for $\xi=1$ the interaction $Q$ becomes infinite. However, we remark that $\xi=1$ represents a very strong interaction in the dark sector, which is not allowed by the observational data; see, for instance, Refs. [39-44]. The interaction (5) has a very special and nice property. For a barotropic equation of state $p_{x}=w_{x} \rho_{x}$, the interaction is linear, while, on the other hand, for any complicated equation of state other than the barotropic equation of state, the interaction (5) represents a nonlinear interaction in the dark sector. Another interesting scenario we observe in the above interaction is as follows. If we the dark energy is assumed to be the cosmological constant, that means $p_{x}=-\rho_{x}$, then the coupling becomes zero $(Q=0)$ even if the coupling parameter is nonzero. That means that, although an interaction exists, the evolution equations do not change. In the present work, we consider that the dark-energy fluid obeys a barotropic equation of state $w_{x}$ and thus the interaction (5) can be recast as

$$
Q=\frac{3}{a}\left(\frac{\xi}{\xi-1}\right) \mathcal{H}\left(1+w_{x}\right) \rho_{x} .
$$

Now, in order to measure the coupling of the interaction in presence of a dark-energy fluid, we consider two distinct possibilities: (A) when the dark-energy equation of state is constant or (B) when the dark-energy equation of state is dynamical. For the dynamical dark energy equation of state, we consider the most well known dark energy parametrization known as Chevallier-Polarski-Linder (CPL) parametrization $[62,63]$, where the equation of state for dark energy is represented by $w_{x}=w_{0}+w_{a}(1-a)$. Here $w_{0}$ is the current value of $w_{x}$, i.e., $w_{0}=w_{x}$ at $a=1$ (we note that the present value of the scale factor has been set to be unity) and $w_{a}=d w_{x} / d a$ at $a=1$.

\section{INTERACTING COSMOLOGY: PERTURBED UNIVERSE}

Now, in order to study the linear perturbations of the interacting dark-energy models, we introduce the most general scalar mode perturbation, defined by the metric [64-66]

$$
\begin{aligned}
d s^{2}= & a^{2}(\tau)\left[-(1+2 \phi) d \tau^{2}+2 \partial_{i} B d \tau d x^{i}\right. \\
& \left.+\left((1-2 \psi) \delta_{i j}+2 \partial_{i} \partial_{j} E\right) d x^{i} d x^{j}\right],
\end{aligned}
$$

where $\phi, B, \psi$, and $E$ are the gauge-dependent scalar perturbations quantities. Let us proceed with the general perturbation equations. We consider the 4-velocity of any fluid (denoted by A) as $u_{A}^{\mu}=a^{-1}\left(1-\phi, \partial^{i} v_{A}\right)$, where $v_{A}$ is the peculiar velocity potential, which in Fourier space is related to the volume expansion $\theta_{A}=-k^{2}\left(v_{A}+B\right)$. Now, since we are considering a coupling between dark matter and dark energy, we have the constraint $\nabla_{\nu} T_{A}^{\mu \nu}=Q_{A}$, where $\sum_{A} Q_{A}=0$ and $T_{A}^{\mu \nu}$ is the usual energy-momentum tensor of the fluid $A$. Now, because of the coupling between the dark sectors, the energy flow and the momentum flow take place in general, thus representing $\tilde{Q}_{A}$ and $F_{A}^{\mu}$ as, respectively, the energy transfer rate and the momentum transfer rate, relative to the 4-velocity $u^{\mu}$, following Refs. $[14,67,68]$, one can write

$$
Q_{A}^{\mu}=\tilde{Q}_{A} u^{\mu}+F_{A}^{\mu},
$$

where $\tilde{Q}_{A}=Q_{A}+\delta Q_{A}, \quad F_{A}^{\mu}=a^{-1}\left(0, \partial^{i} f_{A}\right)$ in which $Q_{A}$ is the background interaction (in fact, $Q_{A}=Q$ ), and $f_{A}$ is the momentum transfer potential. The perturbed interaction assumes $Q_{A}^{0}=-a\left[Q_{A}(1+\phi)+\delta Q_{A}\right]$ and $Q_{A}^{i}=$ $a \partial^{i}\left[Q_{A}(v+B)+f_{A}\right]$, and, finally, the continuity as well as the Euler equations can, respectively, be written as

$$
\begin{aligned}
\delta_{A}^{\prime}+ & 3 \mathcal{H}\left(c_{s A}^{2}-w_{A}\right) \delta_{A}+9 \mathcal{H}^{2}\left(1+w_{A}\right)\left(c_{s A}^{2}-c_{a A}^{2}\right) \frac{\theta_{A}}{k^{2}} \\
& +\left(1+w_{A}\right) \theta_{A}-3\left(1+w_{A}\right) \psi^{\prime}+\left(1+w_{A}\right) k^{2}\left(B-E^{\prime}\right) \\
= & \frac{a}{\rho_{A}}\left(\delta Q_{A}-Q_{A} \delta_{A}\right)+\frac{a Q_{A}}{\rho_{A}}\left[\phi+3 \mathcal{H}\left(c_{s A}^{2}-c_{a A}^{2}\right) \frac{\theta_{A}}{k^{2}}\right],
\end{aligned}
$$

$$
\begin{aligned}
\theta_{A}^{\prime} & +\mathcal{H}\left(1-3 c_{s A}^{2}\right) \theta_{A}-\frac{c_{s A}^{2}}{1+w_{A}} k^{2} \delta_{A}-k^{2} \phi \\
& =\frac{a}{\left(1+w_{A}\right) \rho_{A}}\left[\left(Q_{A} \theta-k^{2} f_{A}\right)-\left(1+c_{s A}^{2}\right) Q_{A} \theta_{A}\right],
\end{aligned}
$$

where we have used the notation $\delta_{A}=\delta \rho_{A} / \rho_{A}$ known as the density contrast and considered $\pi_{A}=0$. The symbols $c_{s A}^{2}$ and $c_{a A}^{2}$, respectively, denote the adiabatic and physical sound velocity, and $\theta=\theta_{\mu}^{\mu}$ is the volume expansion scalar. We note that, in order to escape from any kind of instabilities, we need to assume $c_{s A}^{2} \geq 0$. Now, for this specific interaction between dark energy and dark matter, the above perturbation equations can be recast as

$$
\begin{aligned}
\delta_{x}^{\prime}= & -\left(1+w_{x}\right)\left(\theta_{x}+\frac{h^{\prime}}{2}\right) \\
& -3 \mathcal{H}\left(c_{s x}^{2}-w_{x}\right)\left[\delta_{x}+3 \mathcal{H}\left(1+w_{x}\right) \frac{\theta_{x}}{k^{2}}\right] \\
& +3 \mathcal{H} \frac{\xi}{\xi-1}\left(1+w_{x}\right)\left[\frac{\theta+h^{\prime} / 2}{3 \mathcal{H}}+3 \mathcal{H}\left(c_{s x}^{2}-w_{x}\right) \frac{\theta_{x}}{k^{2}}\right],
\end{aligned}
$$




$$
\begin{aligned}
& \theta_{x}^{\prime}=-\mathcal{H}\left(1-3 c_{s x}^{2}\right) \theta_{x}+\frac{c_{s x}^{2}}{\left(1+w_{x}\right)} k^{2} \delta_{x} \\
&+3 \mathcal{H} \frac{\xi}{\xi-1}\left[\theta_{c}-\left(1+c_{s x}^{2}\right) \theta_{x}\right], \\
& \delta_{c}^{\prime}=-\left(\theta_{c}+\frac{h^{\prime}}{2}\right)+3 \mathcal{H} \frac{\xi}{\xi-1}\left(1+w_{x}\right) \frac{\rho_{x}}{\rho_{c}} \\
& \times\left(\delta_{c}-\delta_{x}-\frac{\theta+h^{\prime} / 2}{3 \mathcal{H}}\right), \\
& \theta_{c}^{\prime}=-\mathcal{H} \theta_{c},
\end{aligned}
$$

where $\delta \mathcal{H} / \mathcal{H}=\left(\theta+h^{\prime} / 2\right) /(3 \mathcal{H})$ is the perturbed Hubble expansion rate [69] in which the prime denotes the derivative with respect to the conformal time.

Now, due to the presence of interaction between dark matter and dark energy, the pressure perturbation equation for dark energy directly includes the interaction rate $Q$, and consequently, the stability of the interaction model becomes sensitive to the specific forms for $Q$. Moreover, the stability of the interaction model is also related to the dark energy equation of state which appears in the pressure perturbation equation for dark energy as [70]

$$
\begin{aligned}
\delta p_{x}= & c_{s x}^{2} \delta \rho_{x} \\
& +3 \mathcal{H} \rho_{x}\left(1+w_{x}\right)\left(c_{s x}^{2}-c_{a x}^{2}\right)\left[1-\frac{a Q}{3 \mathcal{H} \rho_{x}\left(1+w_{x}\right)}\right] \frac{\theta_{x}}{k^{2}},
\end{aligned}
$$

from which one can define the doom factor [70]: $d \equiv$ $-a Q /\left[3 \mathcal{H} \rho_{x}\left(1+w_{x}\right)\right]$ which analyzes the stability of the concerned interaction model by its sign and the stability is achieved for $d \leq 0$. Now, for any interaction $Q=\xi \bar{Q}$ $(\bar{Q}>0$, but $\xi$, the coupling parameter of the interaction $Q$, is unrestricted in sign), the stability criterion (i.e., $d \leq 0$ ) provides a restriction on the parameters space as either (i) $\xi \geq 0$ and $\left(1+w_{x}\right) \geq 0$ or (ii) $\xi \leq 0$ and $\left(1+w_{x}\right) \leq 0$. That means in order to test the stability of any interaction two separate regions must be investigated. Now, if the interaction contains a factor $\left(1+w_{x}\right)$, that means if $Q=$ $\xi\left(1+w_{x}\right) \bar{Q}$ (where similarly $\bar{Q}>0$ ), the doom factor becomes $d \equiv-a \xi \bar{Q} /\left(3 \mathcal{H} \rho_{x}\right)$, and it shows that the stability of the interaction is now only characterized by the sign of $\xi$ only. That means the inclusion of the factor $\left(1+w_{x}\right)$ in the interaction releases the prior on $w_{x}$, which is a beautiful result because now the restriction on the parameter $w_{x}$ is withdrawn, and hence one can test the stability of such interaction models for all $w_{x}$. This observation has been noticed in a recent paper [71]; however, the current work is different. Here, we do not need to include the extra factor $\left(1+w_{x}\right)$ because the interaction that we propose in this work already contains such a factor and hence the prior on the dark-energy equation of state, $w_{x}$, automatically released. To show this, we consider the doom factor for the current interaction (5), which becomes $d=\xi /(1-\xi)$. Since for $d \leq 0$ the perturbation evolutions are stable, we conclude that the present interacting dark-energy model will be stable if either $\xi \geq 1$ or $\xi \leq 0$. However, the possibility $\xi \geq 1$ refers to a strong interaction in the dark sector, which is not allowed by the present observational data, so we confine our discussions over $\xi \leq 0$.

Finally, we close this section with the introduction of growth of matter perturbations. Under the assumption that dark energy does not cluster on the sub-Hubble scale [14], one can safely neglect the velocity perturbations of $\delta_{x}=\delta \rho_{x} / \rho_{x}$. This assumption is genuine because during the matter dominated era the acting of dark energy should be subdominant. Therefore, using Eq. (13), one can derive the second-order differential equation for the density perturbations of pressureless dark matter,

$$
\begin{aligned}
\delta_{c}^{\prime \prime}+ & {\left[1-3 \frac{\xi}{\xi-1}\left(1+w_{x}\right) \frac{\rho_{x}}{\rho_{c}}\right] \mathcal{H} \delta_{c}^{\prime} } \\
= & \frac{3}{2} \mathcal{H}^{2} \Omega_{b} \delta_{b}+\frac{3}{2} \mathcal{H}^{2} \Omega_{c} \delta_{c}\left\{1+2 \frac{\xi}{\xi-1}\left(1+w_{x}\right) \frac{\rho_{t} \rho_{x}}{\rho_{c}} \frac{\rho_{c}}{\rho_{c}}\right. \\
& \times\left[\frac{\mathcal{H}^{\prime}}{\mathcal{H}^{2}}+1-3 w_{x}+3 \frac{\xi}{\xi-1}\left(1+w_{x}\right)\left(1+\frac{\rho_{x}}{\rho_{c}}\right)\right. \\
& \left.\left.+\frac{w_{x}^{\prime}}{\mathcal{H}\left(1+w_{x}\right)}\right]\right\},
\end{aligned}
$$

where $\mathcal{H}^{2}=\left(8 \pi G a^{2} / 3\right) \rho_{t}$ and $\rho_{t}$, is the total energy density of the universe, which means $\rho_{t}=\rho_{r}+$ $\rho_{b}+\rho_{c}+\rho_{x}$. The absence of interaction directs the equation (16) into $\delta_{m}^{\prime \prime}+\mathcal{H} \delta_{m}^{\prime}=4 \pi G \rho_{m} \delta_{m} \quad\left(\rho_{m}=\rho_{c}+\rho_{b}\right)$. Certainly, it is evident that the evolution of $\delta_{c}$ without any interaction is affected in presence of any interaction as shown in Eq. (16). One natural choice is to measure the expansion history due to the interaction, denoted by $\mathcal{H}_{\text {eff }}$, and it can be calculated as

$$
\frac{\mathcal{H}_{\text {eff }}}{\mathcal{H}}=1-\frac{3 \xi}{\xi-1}\left(1+w_{x}\right) \frac{\rho_{x}}{\rho_{c}},
$$

which shows that for $\xi=0$, when there is no interaction between dark matter and dark energy, $\mathcal{H}_{\text {eff }}=\mathcal{H}$. ${ }^{1}$ Moreover, one can also see that for $w_{x}=-1$, when cosmological constant interacts with dark matter, $\mathcal{H}_{\text {eff }}=\mathcal{H}$. While on the other hand, there is another quantity, namely, the gravitational constant, $G$, that is also modified in the presence of the interaction. We denote the modified gravitational constant as $G_{\text {eff }}$, defined by

$$
\begin{aligned}
\frac{G_{\text {eff }}}{G}= & 1+\frac{2 \xi}{\xi-1}\left(1+w_{x}\right) \frac{\rho_{t}}{\rho_{c}} \frac{\rho_{x}}{\rho_{c}}\left[\frac{\mathcal{H}^{\prime}}{\mathcal{H}^{2}}+1-3 w_{x}\right. \\
& \left.+\frac{3 \xi}{\xi-1}\left(1+w_{x}\right)\left(1+\frac{\rho_{x}}{\rho_{c}}\right)+\frac{w_{x}^{\prime}}{\mathcal{H}\left(1+w_{x}\right)}\right] .
\end{aligned}
$$

\footnotetext{
${ }^{1}$ We one may note that $\frac{\mathcal{H}_{\text {eff }}}{\mathcal{H}}=\frac{H_{\text {eff }}}{H}$.
} 
It is easy to see that in the absence of any coupling, i.e., when $\xi=0, G_{\text {eff }}=G$, as expected. Also, for the case of an interacting cosmological constant, this equality holds well. From the evolution of $\mathcal{H}_{\text {eff }} / \mathcal{H}$ and $G_{\text {eff }} / G$, one can quantify the actual deviation of $\mathcal{H}$ and $G$, when interaction in the dark sectors is considered. Lastly. we would like to remark on the growth rate of dark matter, which is $f_{c} \equiv \frac{d}{d \ln a}\left(\ln \delta_{c}\right)$. Since the Euler equation is modified in the presence of the interaction, the dark matter may not follow the geodesics [72]. So, this quantity also plays an important role in measuring the deviation of the interacting models from the noninteracting one.

\section{DATA AND THE METHODOLOGY}

In this section, we shall shortly describe the astronomical data that have been used to constrain both the interacting dark-energy models and the methodology that we use to constrain the interacting scenarios:

(i) $\mathrm{CMB}$ data: Cosmic microwave background (CMB) data provide tight constraints on the cosmological models. Here, we take the CMB data from the latest observations by the Planck team $[46,47]$ that combine the likelihoods $C_{l}^{T T}, C_{l}^{E E}$, and $C_{l}^{T E}$ in addition to low- $l$ polarization. We shall denote these data by Planck TT, TE, EE + lowTEB as denoted in Ref. [1].

(ii) BAO data: The baryon acoustic oscillation (BAO) data are also powerful to probe the nature of dark energy. In our analysis, we use the estimated ratio $r_{s} / D_{V}$ as a "standard ruler" in which $r_{s}$ is the comoving sound horizon at the baryon drag epoch and $D_{V}$ is the effective distance determined by the angular diameter distance $D_{A}$ and Hubble parameter $H$ as $D_{V}(z)=\left[(1+z)^{2} D_{A}(a)^{2} \frac{z}{H(z)}\right]^{1 / 3}$. Three different measurements such as $r_{s}\left(z_{d}\right) /$ $D_{V}(z=0.106)=0.336 \pm 0.015$ from six-degree Field Galaxy Redshift Survey (6dFGRS) data [48], $r_{s}\left(z_{d}\right) / D_{V}(z=0.35)=0.1126 \pm 0.0022$ from Sloan Digital Sky Survey (SDSS) Data Release (DR) 7 data [49], and $r_{s}\left(z_{d}\right) / D_{V}(z=0.57)=$ $0.0732 \pm 0.0012$ from the SDSS DR9 [50] have been considered.

(iii) Joint Light Curves (JLA) sample: The first data set that proved the existence of some dark-energy fluid in the universe sector is the Supernovae Type Ia (SNIa). In the current analysis, we use the latest compilation of the SNIa, namely, the JLA sample [51] that constrains 740 SNIa in the redshift range $z \in[0.01,1.30]$.

(iv) Weak lensing (WL) data: We add the weak gravitational lensing data from blue galaxy sample complied from Canada-France-Hawaii Telescope Lensing Survey [52,53] to other data sets.

(v) Redshift space distortion (RSD) data: We use RSD data from different observational surveys from
2dFGRS [54], the WiggleZ [55], the SDSS LRG [56], the BOSS CMASS [57], the 6dFGRS [58], and the VIPERS [59]. For the measured values of RSD, we refer Table I of Ref. [16].

(vi) Cosmic chronometers (CC) data: We add the Hubble parameter measurements to our analysis. To measure the Hubble parameter values at different redshifts, we use the cosmic chronometers data that have been recently released in the redshift interval $0<z<2$ [60], and such CC data are very powerful to probe the nature of dark energy due to their model independent character. For a detailed analysis of the data and the methodology, we refer to Ref. [60].

(vii) Local Hubble constant: Finally, we add the local Hubble constant value $H_{0}=73.02 \pm 1.79 \mathrm{~km} / \mathrm{s} /$ Mpc obtained with $2.4 \%$ precision by the Riess et al. [61]. We denote it by R16.

Now, to constrain the present interacting models, we use the likelihood $\mathcal{L} \propto e^{-\chi_{\text {tot }}^{2} / 2}$. Here, $\chi_{\text {tot }}^{2}=\sum_{i} \chi_{i}^{2}$, and $i$ runs over the all data sets that we use, which means CMB (Planck TT, TE, EE + lowTEB), BAO, BAO, WL, RSD, $\mathrm{CC}$, and R16. Then we use the Cosmoc [73], a Markov chain Monte Carlo simulation to extract the cosmological parameters associted with the model. Corresponding to each interacting model, our parameter space is increased in compared to the $\Lambda \mathrm{CDM}$ model with minimum dimensional parameter space; see Ref. [74] for more discussions in this direction. For the interacting model with a constant equation of state in dark energy, $w_{x}$, we have the eightdimensional parameters space

TABLE I. The table summarizes the constraints on the free parameters of IDE 1 (IDE with constant $w_{x}$ ) for the joint analysis Planck TT, TE, EE + lowTEB + BAO + JLA + RSD+ $\mathrm{WL}+\mathrm{CC}+\mathrm{R} 16$. Here, we mention that $\Omega_{m 0}=\Omega_{c 0}+\Omega_{b 0}$.

\begin{tabular}{lccc}
\hline \hline Parameters & Priors & Mode $\pm 1 \sigma \pm 2 \sigma$ & Best fit \\
\hline$\Omega_{c} h^{2}$ & {$[0.01,0.99]$} & $0.11773_{-0.00128-0.00273}^{+0.00143+0.00268}$ & 0.11725 \\
$\Omega_{b} h^{2}$ & {$[0.005,0.1]$} & $0.02228_{-0.00017-0.00029}^{+0.00016+0.00032}$ & 0.02230 \\
$100 \theta_{M C}$ & {$[0.5,10]$} & $1.04068_{-0.00031-0.00064}^{+0.00033+0.00062}$ & 1.04051 \\
$\tau$ & {$[0.01,0.8]$} & $0.06593_{-0.01618-0.03060}^{+0.01599+0.03192}$ & 0.07098 \\
$n_{s}$ & {$[0.5,1.5]$} & $0.97692_{-0.00404-0.00777}^{+0.00389+0.00787}$ & 0.98041 \\
$\ln \left(10^{10} A_{s}\right)$ & {$[2.4,4]$} & $3.06864_{-0.03129-0.05305}^{+0.03149+0.06361}$ & 3.07831 \\
$w_{x}$ & {$[-2,0]$} & $-1.01014_{-0.02583-0.05786}^{+0.03101+0.05702}$ & -1.02844 \\
$\xi$ & {$[-1,0]$} & $-0.00857_{-0.04001-0.23056}^{+0.00857+0.00857}$ & -0.02682 \\
$\Omega_{m 0}$ & $\ldots$ & $0.29674_{-0.00877-0.01902}^{+0.00959+0.01877}$ & 0.29510 \\
$\sigma_{8}$ & $\ldots$ & $0.81393_{-0.01411-0.02289}^{+0.01165+0.02510}$ & 0.819897 \\
$H_{0}$ & $\ldots$ & $68.60277_{-0.82512-1.55987}^{+0.79166+1.63707}$ & 68.82080 \\
$\chi_{\min }^{2}$ & $\ldots$ & $\ldots$ & 13720.258 \\
\hline \hline
\end{tabular}




$$
\mathcal{P}_{1} \equiv\left\{\Omega_{b} h^{2}, \Omega_{c} h^{2}, \Theta_{S}, \tau, w_{x}, \xi, n_{s}, \log \left[10^{10} A_{S}\right]\right\},
$$

while for the interacting model with dynamical darkenergy equation of state $w_{x}=w_{0}+w_{a}(1-a)$, the ninedimensional parameters space

$\mathcal{P}_{2} \equiv\left\{\Omega_{b} h^{2}, \Omega_{c} h^{2}, \Theta_{S}, \tau, w_{0}, w_{a}, \xi, n_{s}, \log \left[10^{10} A_{S}\right]\right\}$

is considered. We note that in both Eqs. (19) and (20), the common parameters, $\Omega_{b} h^{2}, \Omega_{c} h^{2}, \Theta_{S}, \tau, n_{s}$, and $A_{S}$, are, respectively, identified as the baryons density, cold darkmatter density, ratio of the sound horizon to the angular diameter distance, optical depth, scalar spectral index, and amplitude of the initial power spectrum, whereas $w_{x}$ and $\xi$ are the model parameters of the parameters space $\mathcal{P}_{1}$, and $w_{0}, w_{a}$, and $\xi$ are the model parameters of the space $\mathcal{P}_{2}$.

\section{RESULTS AND INTERPRETATIONS}

We present a detailed analysis of the interacting scenario considering that the equation of state for dark energy, i.e., $w_{x}$, could be either constant or dynamical. For convenience, we rename the interacting scenario with a constant equation of state in DE as IDE 1, while the interacting scenario with dynamical DE assuming the CPL parametrization by IDE 2 . In the following subsections, we separately discuss both the scenarios.

\section{A. IDE 1: Constant $w_{x}$}

For the constant dark-energy equation of state, we have analyzed the interacting model with the combined analysis Planck TT, TE, EE + lowTEB + BAO + JLA + RSD + $\mathrm{WL}+\mathrm{CC}+\mathrm{R} 16$. In Table I, we summarize the observational constraints of this scenario for these combined data,

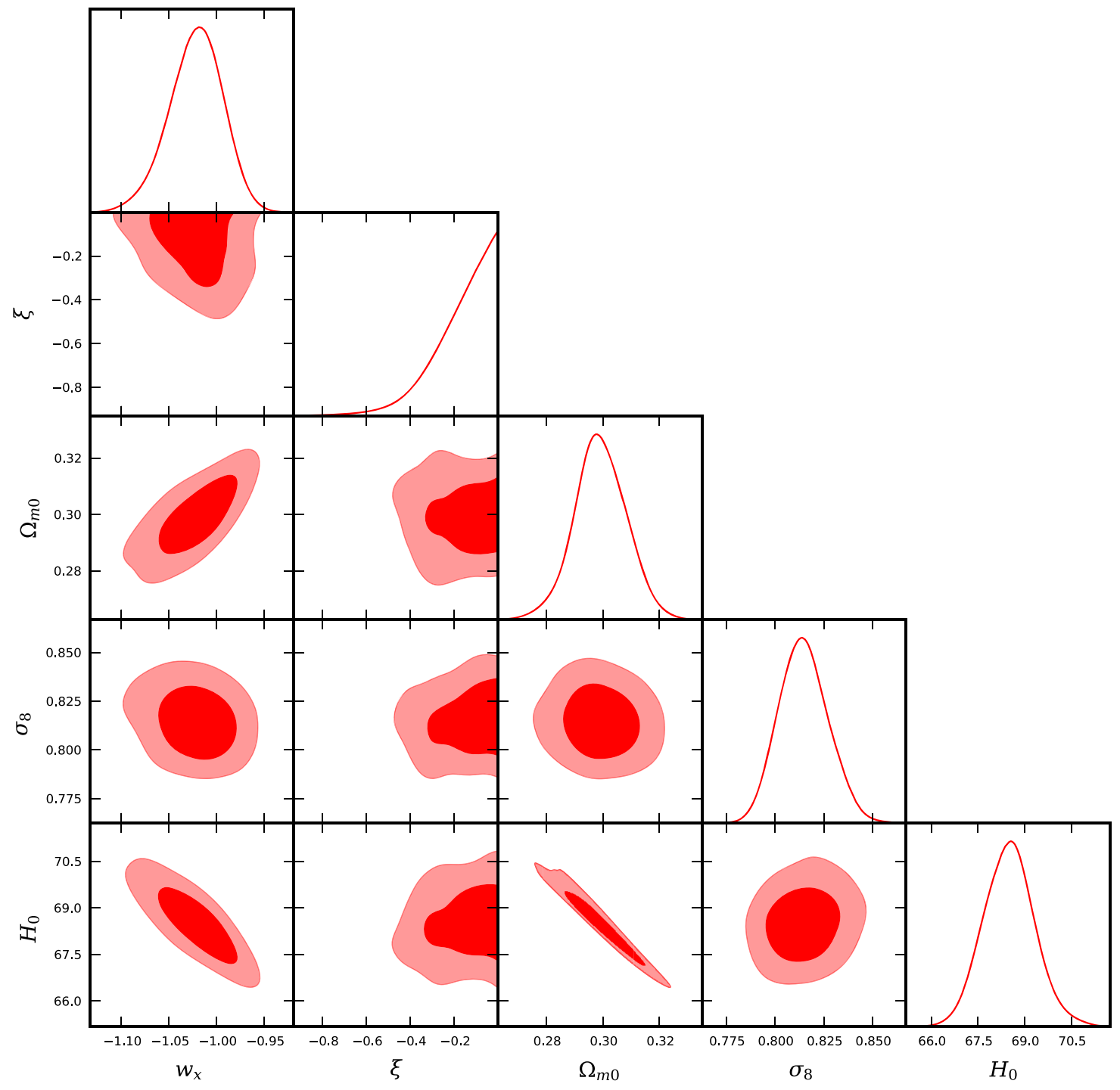

FIG. 1. $\quad 68.3 \%$ and $95.4 \%$ confidence-level contour plots for different combinations of the free parameters of IDE 1 have been shown for the combined analysis Planck TT, TE, EE + lowTEB + BAO + JLA + RSD + WL + CC + R16. 

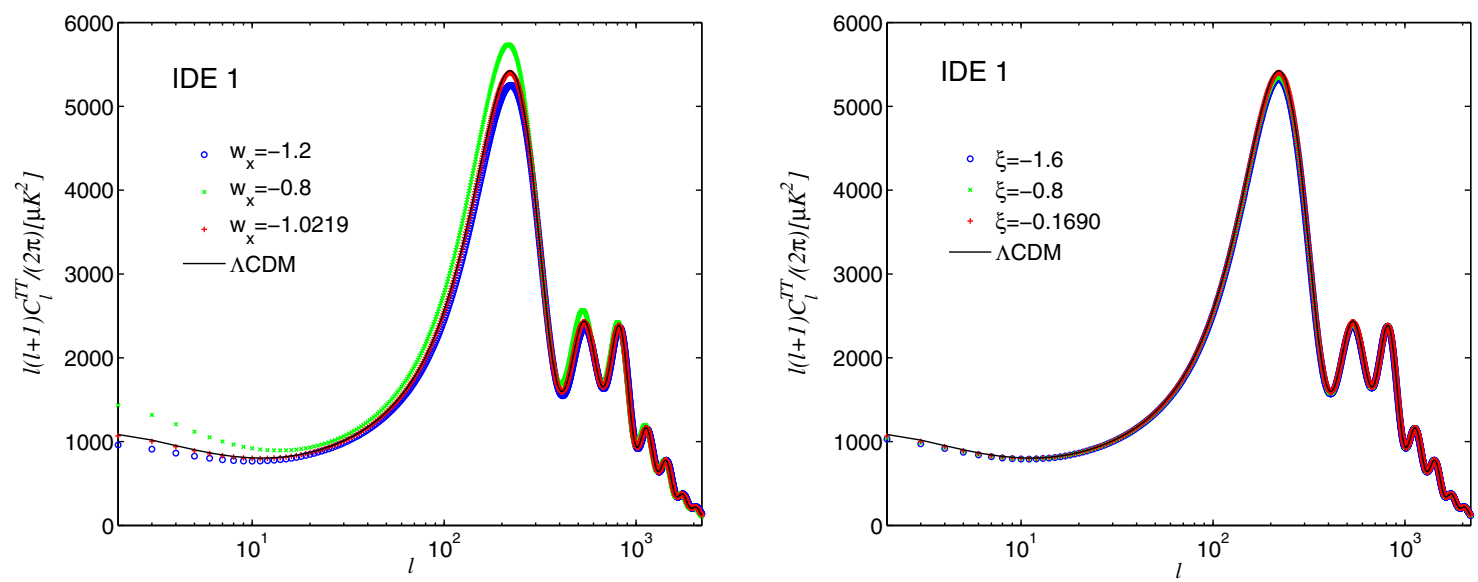

FIG. 2. The plots show the angular CMB temperature anisotropy spectra for IDE 1 over the standard $\Lambda$ CDM cosmology using the joint analysis data Planck TT, TE, EE + lowTEB + BAO + JLA + RSD + WL + CC + R16. In the left panel, we have varied the constant equation of state $w_{x}$, while the right panel stands for different values of the coupling parameter $\xi$. We note that the curves in the right panel are so close to each other that they are practically indistinguishable from each other.

and the corresponding contour plots at $68.3 \%(1 \sigma)$ and $95.4 \%(2 \sigma)$ confidence levels for different combinations of the model parameters along with the one-dimensional posterior distributions of the model parameters have been displayed in Fig. 1.

From our analysis, it is evident that for IDE 1 the coupling parameter as well as the effective coupling parameter are always nonzero. That means the observational data cannot strictly rule out the possibility of a noninteracting scenario. However, within the $1 \sigma$ confidence level, $\xi=0$ is compatible according to the present observational data. Additionally, it is interesting to note that the best-fit value as well as the mode value of the equation of state of DE crosses the phantom divide line; however, the analysis also tells us that within $1 \sigma$ confidence-level $w_{x}=-1$ is consistent with the current astronomical data. Thus, within the $1 \sigma$ confidence region, this interaction model can mimic the $\Lambda$ cosmology. To characterize the large-scale behavior of the IDE models, in Fig. 2, we display the angular CMB temperature anisotropy spectra compared to the $\Lambda \mathrm{CDM}$ cosmology. In particular, in the left panel of Fig. 2, we show the angular CMB temperature anisotropy spectra for different values of the equation of state of DE, and in the right panel of Fig. 2, we show the temperature anisotropy of the angular CMB spectra for different values of the coupling parameter. From the left panel of Fig. 2 we see that the IDE 1 with $w_{x}<-1$ does not deviate much from the $\Lambda$ CDM cosmology. The deviation is very clear when $w_{x}>-1$. However, from the right panel of Fig. 2, we see that the small or large coupling basically does not indicate any significant deviation from the $\Lambda$ CDM cosmology. This is a surprising result. From the left panel of Fig. 2, we see that if $w_{x}$ increases (i.e., if $w_{x}$ becomes more quintessential) then we find the deviation in the $\mathrm{CMB}$ spectra from that of the standard $\Lambda$ CDM cosmology. Further, in Fig. 3, we depict the evolution of the modified Hubble function $\mathcal{H}_{\text {eff }}$ and the modified gravitational constant $G_{\text {eff }}$ in the presence of the coupling between the dark sectors. The plots in Fig. 3 clearly show that as the magnitude of the coupling parameter increases the deviation
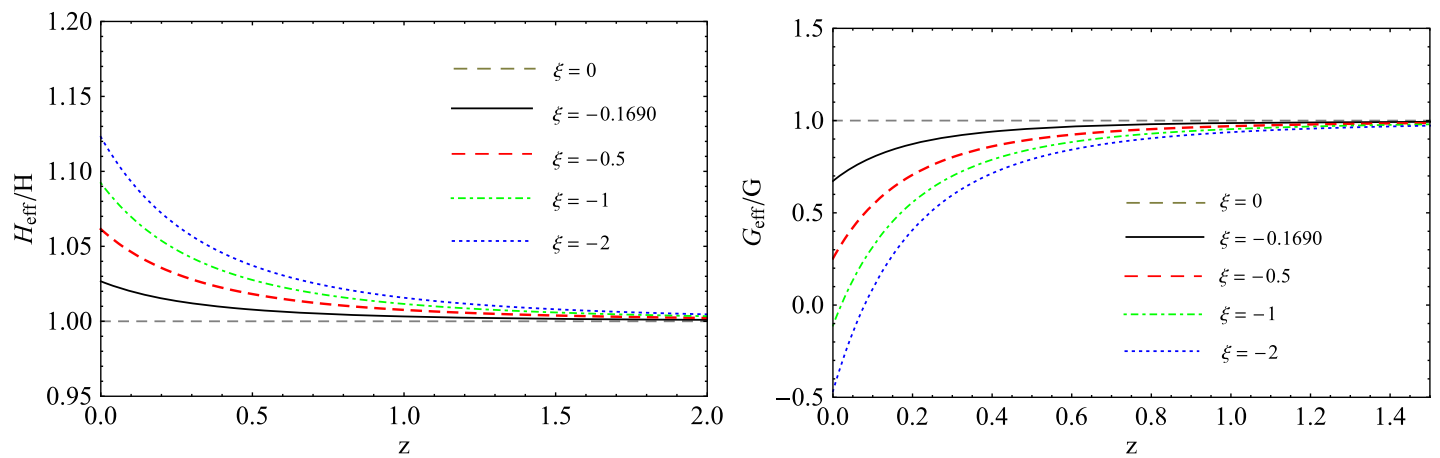

FIG. 3. For different coupling parameters, we show the evolutions of $\mathcal{H}_{\text {eff }} / \mathcal{H}$ (left panel) and $G_{\text {eff }} / G$ (right panel) for the interacting dark-energy model with constant $w_{x}$. The deviation is measured from the noninteracting scenario (i.e., $\left.\xi=0\right)$ and also from the mean value of $\xi=-0.1690$ obtained from the combined analysis Planck TT, TE, EE + lowTEB $+\mathrm{BAO}+\mathrm{JLA}+\mathrm{RSD}+\mathrm{WL}+\mathrm{CC}+\mathrm{R} 16$. 


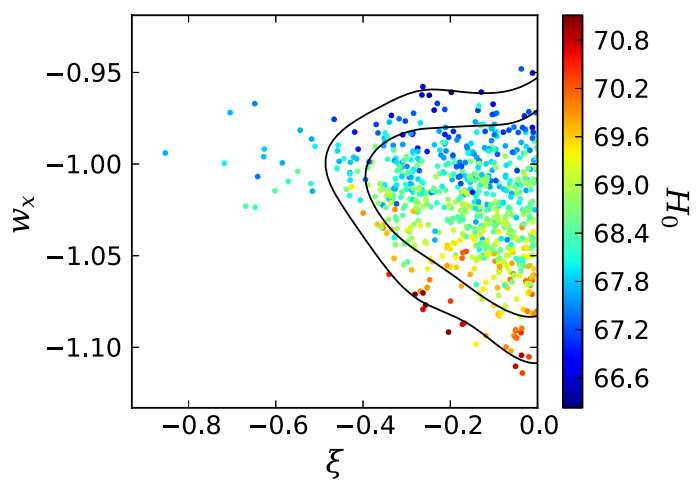

FIG. 4. Markov chain Monte Carlo samples in the $\left(w_{x}, \xi\right)$ plane colored by the Hubble constant value $H_{0}$ for IDE 1 analyzed with the combined analysis Planck TT, TE, EE + lowTEB + BAO + $\mathrm{JLA}+\mathrm{RSD}+\mathrm{WL}+\mathrm{CC}+\mathrm{R} 16$.

of both the modified Hubble function and the gravitational constant increases from that of the noninteracting $w_{x} \mathrm{CDM}$ model.

Finally, we close our analysis with an interesting observation reflected in Fig. 4, which contains the twodimensional marginalized posterior distribution for the parameters $\left(w_{x}, \xi\right)$. The sample points have been taken from the Markov chain Monte Carlo analysis, and they have been colored by the values of $H_{0}$. From this figure, we see that the higher values of $H_{0}$ favor the phantom dark energy, while for the lower values of $H_{0}$, the quintessential dark energy is recommended. Moreover, a shifting of the dark-energy equation of state from its quintessential to phantom behavior is observed as $H_{0}$ decreases. This is one of the interesting observations in this work.

\section{B. IDE 2: Dynamical $w_{x}$}

For the interacting DE with the dynamical equation of state (IDE 2), we present the observational constraints in Table II using the same combined analysis Planck TT, TE, $\mathrm{EE}+$ lowTEB + BAO + JLA + RSD + WL + CC + R16. The corresponding contour plots at $68.3 \%$ and $95.4 \%$ confidence regions for different combinations of the free parameters of this scenario plus the one-dimensional posterior distribution of the free parameters have been shown in Fig. 5. Our analysis shows that the mode value as well as the best-fit values of the present dark-energy equation of state have phantom behavior. However, from the observational data, one can infer that, within $1 \sigma$ confidence level, $w_{0}=-1$ is compatible. The coupling parameter $\xi$ as seen from Table II is nonzero, which signals a noninteracting cosmological scenario, while within $1 \sigma$ confidence level, $\xi=0$ is also consistent with the present observational data. That means that within $1 \sigma$ confidence level the interacting model is indistinguishable from $\Lambda$ cosmology.

On the other hand, from the CMB spectra (see Fig. 6), we observe similar results as we find in IDE 1 . That means that the CMB spectra do not show any significant variation for
TABLE II. Summary of the constraints on the free parameters of IDE 2 (IDE with dynamical $w_{x}$ ) using the joint analysis Planck $\mathrm{TT}, \mathrm{TE}, \mathrm{EE}+$ lowTEB + BAO + JLA + RSD + WL + CC + R16. Here, we note that $\Omega_{m 0}=\Omega_{c 0}+\Omega_{b 0}$.

\begin{tabular}{lccc}
\hline \hline Parameters & Priors & Mode $\pm 1 \sigma \pm 2 \sigma$ & Best fit \\
\hline$\Omega_{c} h^{2}$ & {$[0.01,0.99]$} & $0.11766_{-0.00119-0.00316}^{+0.00158+0.00301}$ & 0.11410 \\
$\Omega_{b} h^{2}$ & {$[0.005,0.1]$} & $0.02224_{-0.00015-0.00029}^{+0.00017+0.00030}$ & 0.02241 \\
$100 \theta_{M C}$ & {$[0.5,10]$} & $1.04061_{-0.00030-0.00063}^{+0.00031+0.00064}$ & 1.04088 \\
$\tau$ & {$[0.01,0.8]$} & $0.06443_{-0.01599-0.03523}^{+0.01633+0.03277}$ & 0.06886 \\
$n_{s}$ & {$[0.5,1.5]$} & $0.97561_{-0.00408-0.00799}^{+0.00410+0.00783}$ & 0.98110 \\
$\ln \left(10^{10} A_{s}\right)$ & {$[2.4,4]$} & $3.06729_{-0.03077-0.06502}^{+0.03133+0.06551}$ & 3.07243 \\
$w_{0}$ & {$[-2,0]$} & $-1.04169_{-0.06889-0.10442}^{+0.05331+0.1093}$ & -1.04824 \\
$w_{a}$ & {$[-3,3]$} & $0.04941_{-0.13556-0.334331}^{+0.19833}$ & 0.11464 \\
$\xi$ & {$[-1,0]$} & $-0.00989_{-0.03969-0.34569}^{+0.00989+0.00989}$ & -0.45436 \\
$\Omega_{m 0}$ & $\ldots$ & $0.29986_{-0.00888-0.01795}^{+0.00987+0.01713}$ & 0.29201 \\
$\sigma_{8}$ & $\ldots$ & $0.82023_{-0.01489-0.02599}^{+0.01291+0.02687}$ & 0.79875 \\
$H_{0}$ & $\ldots$ & $68.42602_{-0.87900-1.51089}^{+0.74521+1.5346}$ & 68.75732 \\
$\chi_{\min }^{2}$ & $\ldots$ & $\ldots$ & 13721.564 \\
\hline \hline
\end{tabular}

different values of the coupling parameter, while for different values of $w_{0}$ and $w_{a}$, we may expect slight variation on the CMB spectra; for instance, in the left panel of Fig. 6, we see that for the high quintessential nature of the dark-energy equation of state a slight difference from the $\Lambda \mathrm{CDM}$ cosmology is observed. Thus, one can assume that if $w_{0}$ increases then the deviation would be prominent. Similarly, in the middle panel of Fig. 6, we see that for $w_{a}>0$ slight variation from $\Lambda \mathrm{CDM}$ cosmology has appeared. In addition to that, like IDE 1, in Fig. 7, we show the evolution of the modified Hubble function $\mathcal{H}_{\text {eff }}$ and the modified gravitational constant $G_{\text {eff }}$ for different values of the coupling parameter. We observe a behavior equivalent to that of IDE 1 . The plots in Fig. 7 clearly show that as the magnitude of the coupling parameter increases the deviation of both the modified Hubble function and the gravitational constant increases from that of the noninteracting $w_{x} \mathrm{CDM}$ model in which $w_{x}$ is dynamical with the form chosen in this figure. However, one may note that the rate of increment of the quantities $\mathcal{H}_{\text {eff }} / \mathcal{H}$ and $G_{\text {eff }} / G$ from the noninteracting models as shown in Fig. 7, is slightly lower in compared to the plots in Fig. 3.

Using the same combined analysis, in Fig. 8, we show two-dimensional marginalized posterior distributions for the parameters $\left(w_{a}, w_{0}\right)$ and $\left(w_{0}, \xi\right)$ colored by the $H_{0}$ sample from the Markov chain Monte Carlo analysis. From the left panel of Fig. 8, we find that for higher values of $H_{0}$ the present value of the dark-energy equation of state, i.e., $w_{0}$, favors the phantom behavior, while for lower values of $H_{0}$, the current value of the dark-energy equation of state, 


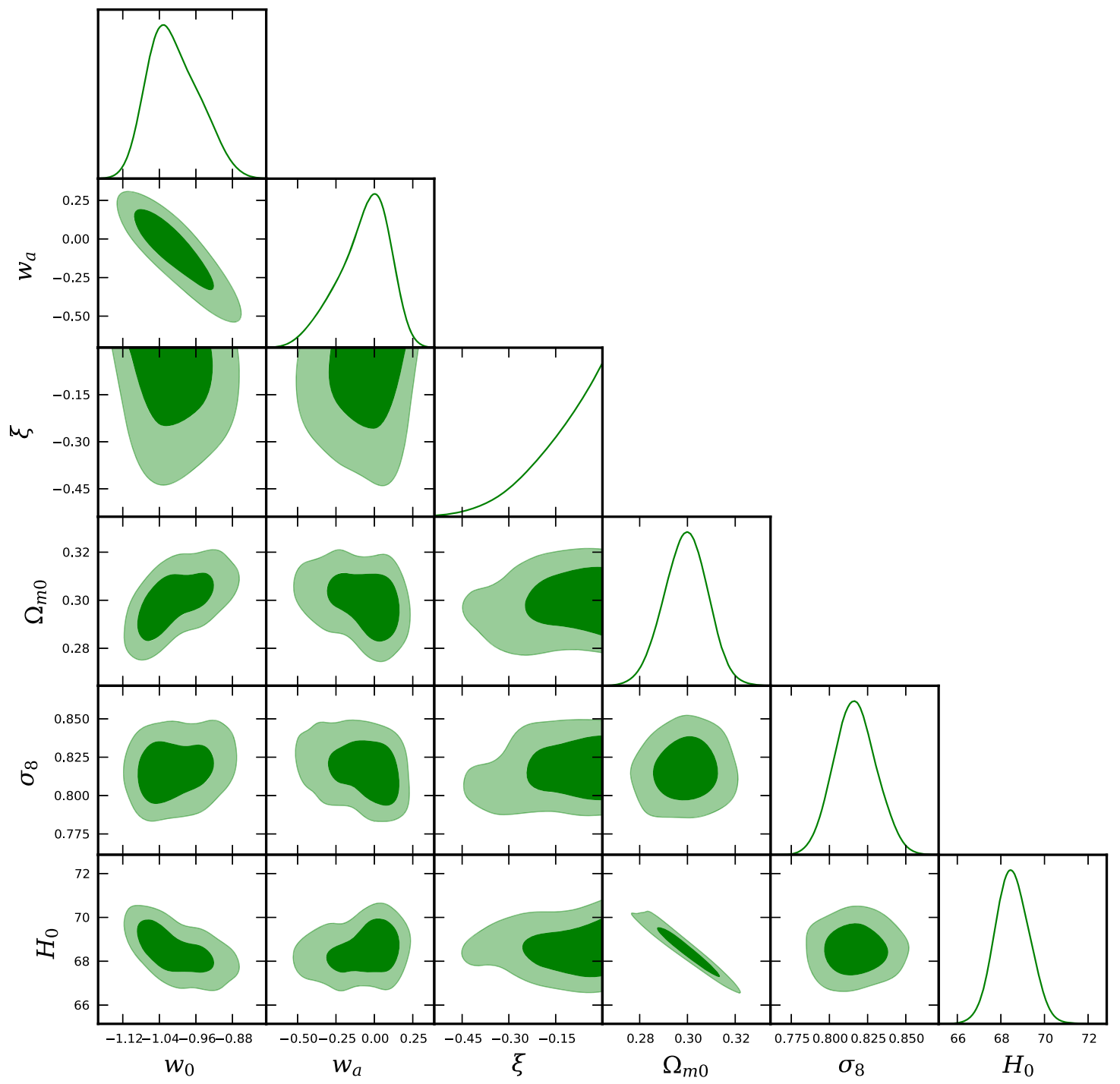

FIG. 5. $68.3 \%$ and $95.4 \%$ confidence-level contour plots for different combinations of the free parameters of IDE 2 have been shown for the combined analysis Planck TT, TE, EE + lowTEB + BAO + JLA + RSD + WL + CC + R16.
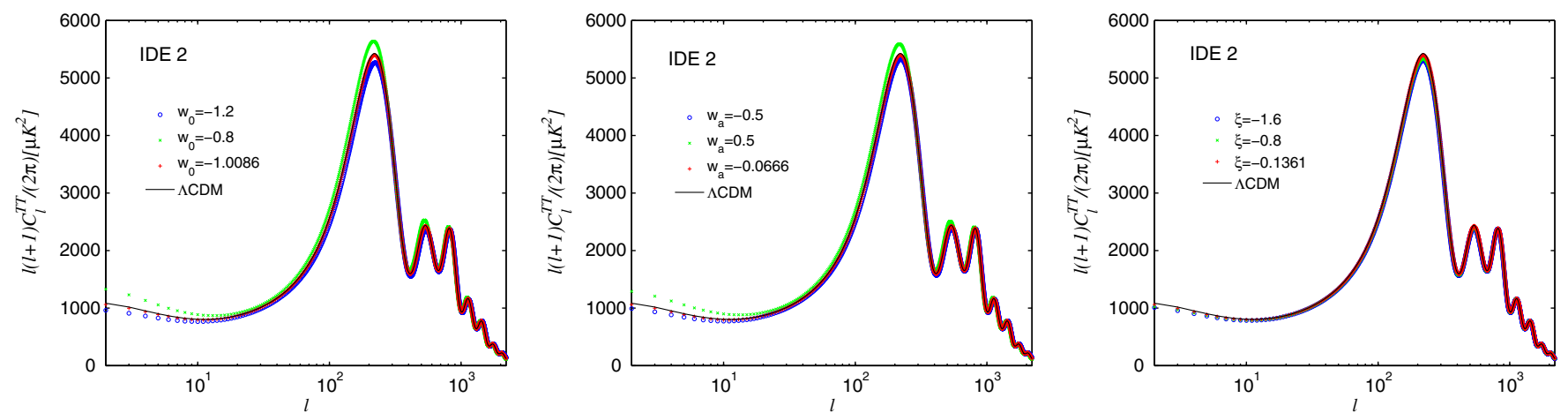

FIG. 6. The plots show the angular CMB termperature anisotropy spectra for IDE 2 over the standard $\Lambda$ CDM cosmology using the joint analysis data Planck TT, TE, EE + lowTEB + BAO + JLA + RSD + WL + CC + R16. In the left panel, we have varied the free parameter $w_{0}$ (i.e., the current value of the equation of state for DE) of the CPL parametrization; in the middle panel, we have varied the parameter $w_{a}$ of the CPL parametrization, and the right panel stands for different values of the coupling parameter $\xi$. We note that, although the curves in the left and middle panels are identified, the curves in the right panel are so close to each other that they are practically indistinguishable from each other. 

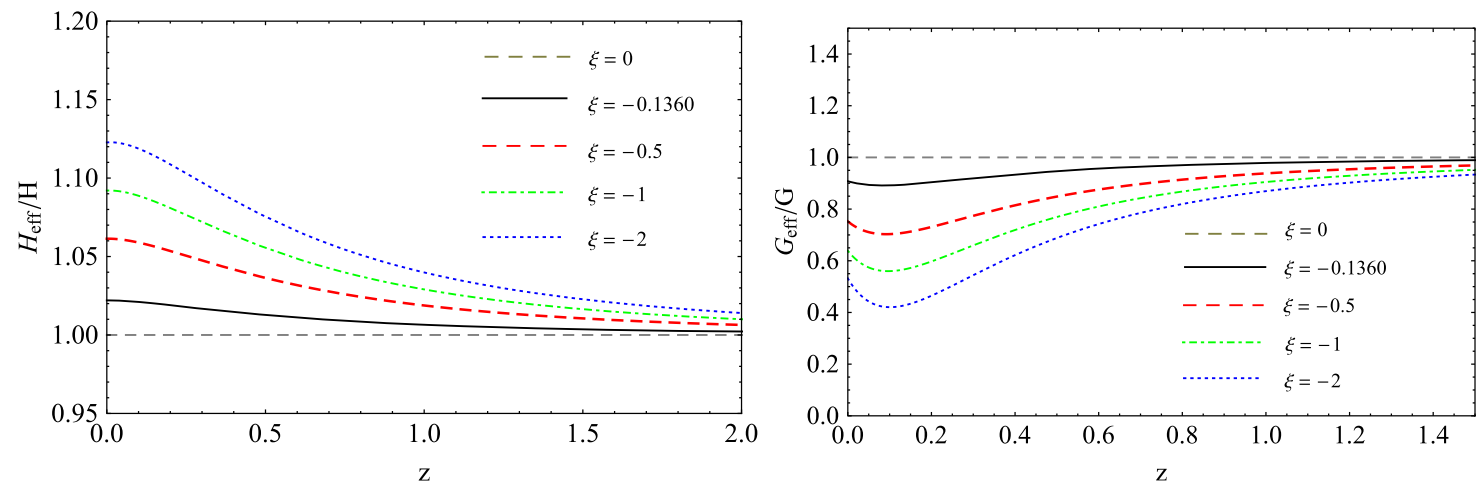

FIG. 7. For the interacting dark-energy model with its dynamical equation of state, $w_{x}=w_{0}+w_{a}(1-a)$, in this plot, using different coupling parameters, we show the evolutions of $\mathcal{H}_{\text {eff }} / \mathcal{H}$ (left panel) and $G_{\text {eff }} / G$ (right panel). The deviation is measured from the noninteracting scenario (i.e., $\xi=0$ ) and also from the mean value of $\xi=-0.1360$ obtained from the combined observational analysis Planck TT, TE, EE + lowTEB + BAO + JLA + RSD + WL + CC + R16.
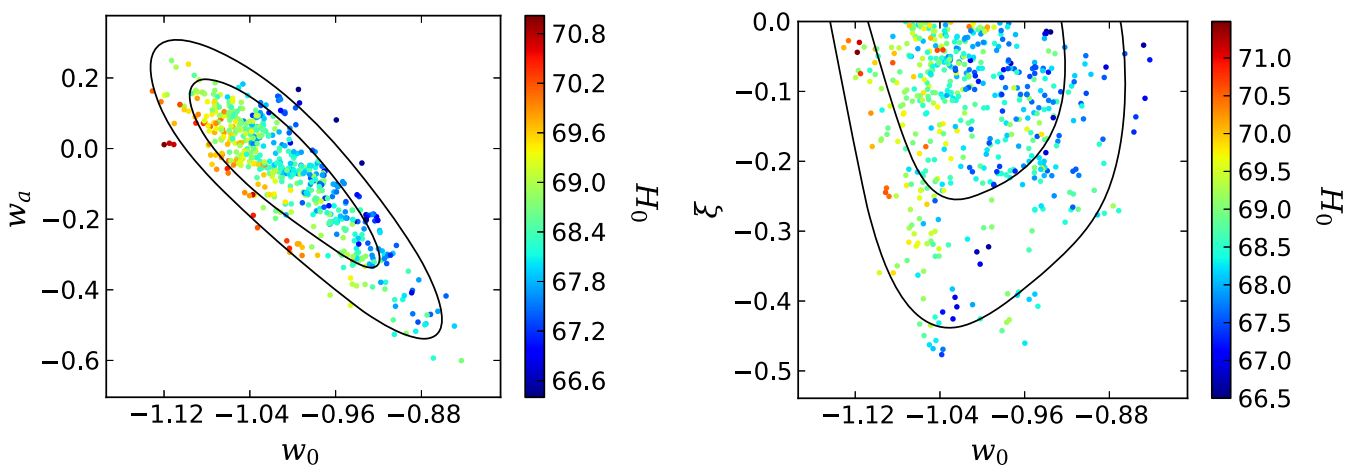

FIG. 8. We show the Markov chain Monte Carlo sample in this figure. In the left panel, we show the two-dimensional posterior distribution for the parameters $\left(w_{0}, w_{a}\right)$ colored by $H_{0}$. In the right panel, the sample space contains the $\left(w_{0}, \xi\right)$ plane colored by $H_{0}$. We note that all the chains have been analyzed with the combined observational data Planck TT, TE, EE + lowTEB $+\mathrm{BAO}+$ $\mathrm{JLA}+\mathrm{RSD}+\mathrm{WL}+\mathrm{CC}+\mathrm{R} 16$.
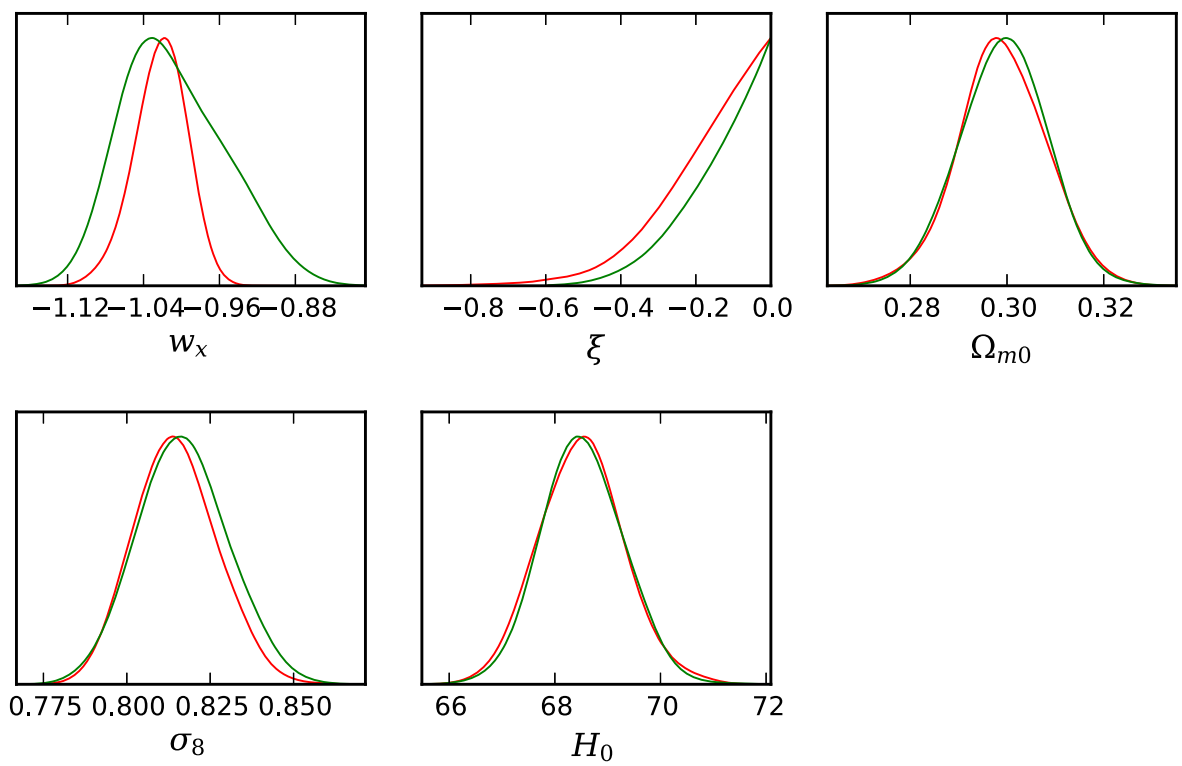

FIG. 9. The figure makes a comparison of the one-dimensional posterior distribution for the free and derived parameters for IDE 1 and IDE 2. The observational data used are Planck TT, TE, EE + lowTEB + BAO + JLA + RSD + WL + CC + R16. We note that in the top left panel we have used only the parameter $w_{x}$, which is also the $w_{0}$ parameter of the dynamical DE identified by $w_{x}=w_{0}+w_{a}(1-a)$. 

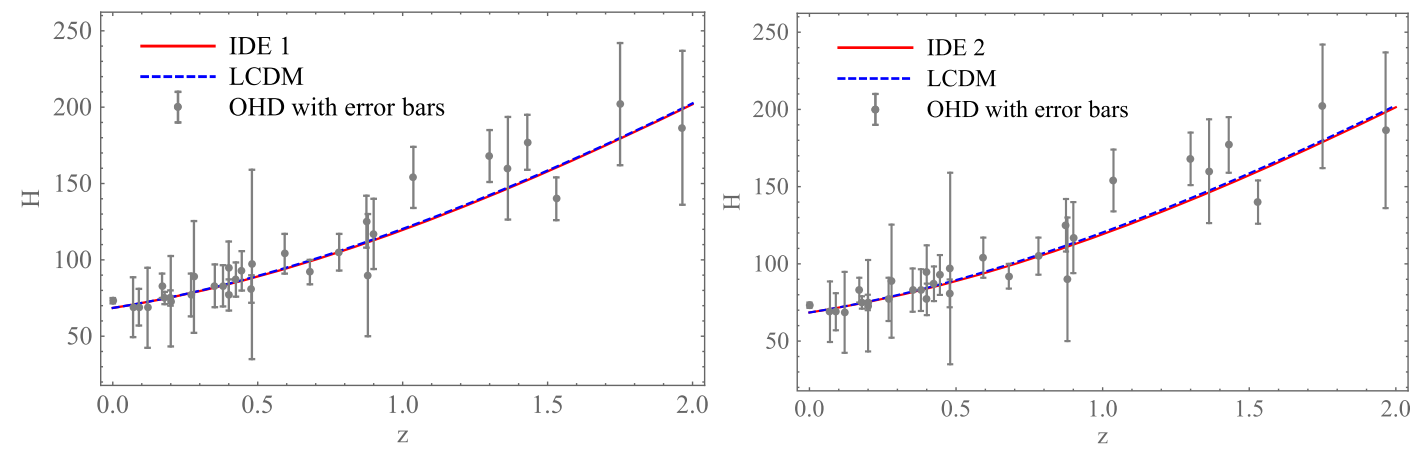

FIG. 10. The evolution of the Hubble parameter for two different IDE models compared to the flat $\Lambda$ CDM model and the error bars of the observed Hubble data are shown. The fitting analysis is the same: Planck TT, TE, EE + lowTEB $+\mathrm{BAO}+\mathrm{JLA}+\mathrm{RSD}+$ $\mathrm{WL}+\mathrm{CC}+\mathrm{R} 16$

i.e., $w_{0}$, shifts toward the quintessence regime. The right panel of Fig. 8 concludes similar behavior obtained from its left panel.

\section{COMPARISONS BETWEEN IDE 1 AND IDE 2 AND WITH OTHER INTERACTIONS}

Interacting models with dynamical dark energy are quite appealing and interesting. In this work, since we consider both constant and time-dependent dark-energy equations of state, it is reasonable to measure their qualitative changes both at the background and perturbative levels. To compare both the models, in Fig. 9, we show the one-dimensional posterior distribution for the free and derived parameters of the interacting models. From the figure, we do not observe any significant changes in the parameters. Although a slight difference appears in $w_{x}$, this does not seem to be a notable one. Moreover, in Fig. 10, we plot the Hubble parameter

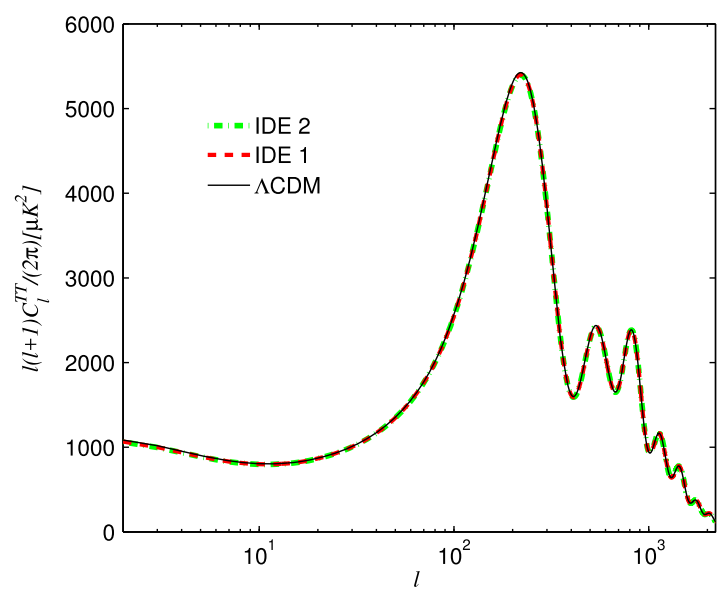

FIG. 11. Using the mean values of the free parameters (from the combined analysis Planck TT, TE, EE + lowTEB + BAO+ $\mathrm{JLA}+\mathrm{RSD}+\mathrm{WL}+\mathrm{CC}+\mathrm{R} 16)$ associated with IDE 1 and IDE 2, in this plot, we show the angular CMB temperature anisotropy spectra to make a comparison of these two interacting scenarios with respect to the $\Lambda \mathrm{CDM}$ cosmology. The curves as seen from the plot are completely indistinguishable. functions for both IDE models with CC + R16. From this figure, we see that both models are very close to the $\Lambda$ cosmology, and in fact, at low redshift, they are quite consistent with the observed data, which are shown in the above plots with their error bars. Therefore, it is clear that at the background level both IDE models are very close to each other and they have considerable closure with the $\Lambda \mathrm{CDM}$ cosmology. It is even very interesting to see that at the perturbative level we do have the same feature.

If we look at the CMB temperature anisotropy spectra in Fig. 11, we see that both IDE 1 and IDE 2 are very close to each other and they are also very close to $\Lambda \mathrm{CDM}$. The slight differences between these models can be detected when the growth rate of cold dark matter is encountered in the picture. We illustrate these slight differences between these two interacting models in Fig. 12. In the left panel of Fig. 12, we display the growth rate of dark matter when the interacting dark energy has a constant equation of state where for different coupling parameter $\xi$, we show a couple of plots. From the plots, we see that as $|\xi|$ increases the growth rate for cold dark matter decreases. Similar behavior is observed when the interacting dark energy has dynamical character. However, for the dynamical DE, we have something more. Here, the growth rate for cold dark matter decreases much more than the growth rate for cold dark matter in the framework of interacting DE with a constant equation of state. However, in summary, one can easily conclude that the models IDE 1 and IDE 2 are statistically very close to each other.

Moreover, we find that the current interaction model is qualitatively different than other interaction models. As of now, in the current literature, different coupled dark-energy models have been introduced and analyzed with the astronomical observations. The most studied and wellknown coupled DE models include the interactions $Q=3 H \xi \rho_{c}, Q=3 H \xi \rho_{x}$, and $Q=3 H \xi\left(\rho_{c}+\rho_{x}\right) \quad(\xi$ is the coupling parameter), and there are many more; see Refs. [5-44]. In this work, we introduce an interaction with the form $Q \propto \dot{\rho}_{x}$, which is different from the others since the variation of the dark-energy density is considered here. 

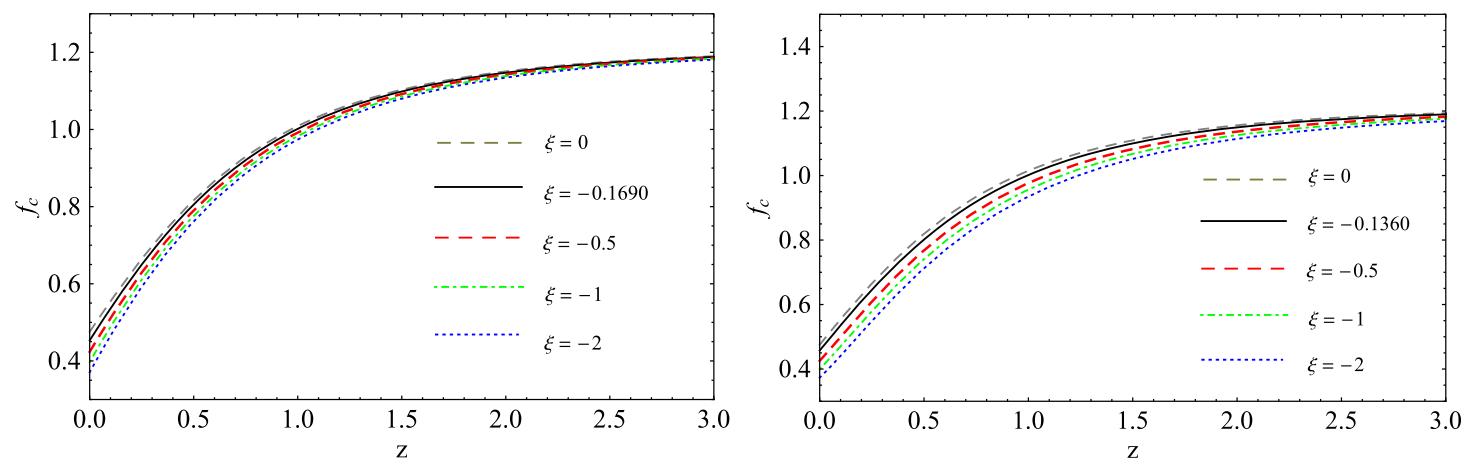

FIG. 12. The evolution of the growth rate of cold dark matter has been shown for both interacting dark-energy models. In the left panel, we show the evolution of the growth rate of cold dark matter for IDE 1, while the right panel contains the same information but for IDE 2. We use the combined observational data Planck TT, TE, EE + lowTEB $+\mathrm{BAO}+\mathrm{JLA}+\mathrm{RSD}+$ $\mathrm{WL}+\mathrm{CC}+\mathrm{R} 16$.

This interaction has a great advantage in compared to some well known interaction models. To clarify such an issue, let us recall the observational bounds on the existing interacting models in the literature. The past analysis of coupled dark-energy models in the large-scale universe reports that the models with $Q=3 H \xi \rho_{c}, Q=3 H \xi \rho_{x}$, and $Q=$ $3 H \xi\left(\rho_{c}+\rho_{x}\right)$ can only be tested for two separate intervals, which means when $\left(w_{x} \geq-1, \xi \geq 0\right)$ or $\left(w_{x} \leq-1, \xi \leq 0\right)$. See Table III where we present some well known interaction models as well as the present interaction model with their testable regions. Clearly, while constraining the above interaction models, we perceive a discontinuity in the parametric space. To remove such a discrepancy in the interaction models, a recent investigation [71] found that the whole parametric space can be tested if a phenomenological factor $\left(1+w_{x}\right)$ is introduced in the interaction function, $Q$. Originally, the hints to introduce such a factor $\left(1+w_{x}\right)$ in the interaction function are motivated from the pressure perturbation of dark energy. Using such a formalism, three interactions have been tested with positive results, namely, $Q=3 H \xi\left(1+w_{x}\right) \rho_{x}, Q=$ $3 H \xi\left(1+w_{x}\right) \rho_{c} \rho_{x} /\left(\rho_{c}+\rho_{x}\right)$, and $Q=3 H \xi\left(1+w_{x}\right) \rho_{x}^{2} / \rho_{c}$ in [71]. However, the current interaction provides something more. We see that the interaction (6) does not need any extra factor $\left(1+w_{x}\right)$ from the outside to test the entire parametric space since it automatically contains such a factor within it. Thus, this interaction differs from other interaction models in this way, and it is worth noting that the entire parameters space can be constrained unlike other interaction models.

\section{TENSION ON $H_{0}$ : ALLEVIATION THROUGH INTERACTING DARK ENERGY}

From the analysis of cosmic microwave background temperature and polarization data by Planck [1], the Hubble constant value is constrained to be $H_{0}=67.27 \pm$ $0.66 \mathrm{~km} \mathrm{~s}^{-1} \mathrm{Mpc}^{-1}$, assuming $\Lambda \mathrm{CDM}$ as the base model, while on the other hand, from the local measurements performed recently by Riess et al. [61], the Hubble constant appears to be $H_{0}=73.24 \pm 1.74 \mathrm{~km} \mathrm{~s}^{-1} \mathrm{Mpc}^{-1}$. This local Hubble constant value is about $3 \sigma$ higher than its prediction by the Planck measurements. The difference appearing in the estimation of $H_{0}$ from the local and global measurements is quite large. It is indeed a very big issue ${ }^{2}$ in the cosmological theory-known as the tension on $H_{0}$.

The interacting dark-energy mechanism carries a reasonable justification to reconcile such a tension that has already been suggested in some recent works [41,44,77]; in Ref. [77], the dark-energy equation of state was constant and allowed to be phantom, while in the other two works, namely, in Refs. [41,44], the dark-energy equations of state were, respectively, constant (other than the cosmological constant) and the cosmological constant.

The current interaction model is completely different compared to the works $[41,44,77]$ because here the model can be tested without any restriction on the dark-energy equation of state as explained earlier. Additionally, here, we investigate the dynamics of the universe for both the constant and dynamical dark-energy equations of state. We also note that for both the models the combined analysis has been fixed to be Planck TT, TE, EE+ lowTEB + BAO + JLA + RSD + WL + CC + R16.

(i) Constant equation of state in $\mathrm{DE}$ and $\mathrm{H}_{0}$ tension: We investigate the tension on $H_{0}$ for four different intervals of the dark-energy equations of state:

(a) We allow a wide range of the dark-energy equation of state from quintessence to phantom, in particular, $w_{x} \in[-1.5,-0.9]$.

(b) We consider the dark-energy equation of state to vary from the cosmological constant boundary to beyond; hence, we fix $w_{x} \in[-2,-1]$.

(c) We consider only phantom dark energy with when $w_{x} \in[-2,-1.01]$.

\footnotetext{
${ }^{2}$ The continuous progress toward more consistent observational measurements could reveal some more information in this regard; see, for instance, Refs. [75,76].
} 
TABLE III. Some known interaction models and the current model with their testable regions.

\begin{tabular}{lll}
\hline \hline Models & \multicolumn{1}{c}{ Coupling } & \multicolumn{1}{c}{ Large-scale stability } \\
\hline Model I & $Q=3 H \xi \rho_{c}$ & either $w_{x} \geq-1$ and $\xi \geq 0$ or $w_{x} \leq-1$ and $\xi \leq 0$ \\
Model II & $Q=3 H \xi \rho_{x}$ & either $w_{x} \geq-1$ and $\xi \geq 0$ or $w_{x} \leq-1$ and $\xi \leq 0$ \\
Model III & $Q=3 H \xi\left(\rho_{c}+\rho_{x}\right)$ & either $w_{x} \geq-1$ and $\xi \geq 0$ or $w_{x} \leq-1$ and $\xi \leq 0$ \\
Present model & $Q=\xi \dot{\rho}_{x}$ & for all $w_{x}$ and $\xi \leq 0$ \\
\hline \hline
\end{tabular}

TABLE IV. For the constant dark-energy equation of state, $w_{x}$, we present the results of the interaction model for four different regions of $w_{x}$. The analysis has been performed for the combined analysis Planck TT, TE, EE + lowTEB $+\mathrm{BAO}+\mathrm{JLA}+$ $\mathrm{RSD}+\mathrm{WL}+\mathrm{CC}+\mathrm{R} 16$. As mentioned earlier, $\Omega_{m 0}=\Omega_{c 0}+\Omega_{b 0}$.

\begin{tabular}{|c|c|c|c|c|}
\hline Parameters & $w_{x} \in[-1.5,-0.9]$ & $w_{x} \in[-2,-1]$ & $w_{x} \in[-2,-1.01]$ & $w_{x} \in[-2,-1.2]$ \\
\hline$\Omega_{c} h^{2}$ & $0.11694_{-0.00128-0.00353}^{+0.00194+0.00302}$ & $0.11702_{-0.00129-0.00311}^{+0.00161+0.00287}$ & $0.11747_{-0.00132-0.00258}^{+0.00136+0.00264}$ & $0.12011_{-0.00121-0.00376}^{+0.00206+0.00332}$ \\
\hline$\Omega_{b} h^{2}$ & $0.02230_{-0.00014-0.00028}^{+0.00014+0.00029}$ & $0.02228_{-0.00014-0.00028}^{+0.00015+0.00028}$ & $0.02226_{-0.00014-0.00029}^{+0.00015+0.00029}$ & $0.02202_{-0.00012-0.00025}^{+0.00013+0.00026}$ \\
\hline $100 \theta_{M C}$ & $1.04070_{-0.00033-0.00060}^{+0.00032+0.00062}$ & $1.04069_{-0.00027-0.00059}^{+0.00029+0.00059}$ & $1.04066_{-0.00030-0.00064}^{+0.00036+0.00060}$ & $1.04023_{-0.00030-0.00064}^{+0.00031+0.00061}$ \\
\hline$\tau$ & $0.06765_{-0.01862-0.03345}^{+0.01602+0.03418}$ & $0.06539_{-0.01700-0.03331}^{+0.01775+0.03354}$ & $0.06178_{-0.01627-0.03299}^{+0.01613+0.03366}$ & $0.03222_{-0.01690-0.02221}^{+0.01031+0.02317}$ \\
\hline$n_{s}$ & $0.97639_{-0.00428-0.00822}^{+0.00419+0.00870}$ & $0.97559_{-0.00390-0.00784}^{+0.00385+0.00842}$ & $0.97491_{-0.00366-0.00804}^{+0.00416+0.00731}$ & $0.96665_{-0.00368-0.00674}^{+0.00339+0.00693}$ \\
\hline $\ln \left(10^{10} A_{s}\right)$ & $3.07394_{-0.03451-0.06387}^{+0.03206+0.06496}$ & $3.07116_{-0.03289-0.06678}^{+0.03186+0.06265}$ & $3.06402_{-0.03180-0.06427}^{+0.03184+0.06524}$ & $3.01185_{-0.03145-0.04863}^{+0.02230+0.05185}$ \\
\hline$w_{x}$ & $-1.02320_{-0.02383-0.05423}^{+0.03135+0.05197}$ & $-1.03245_{-0.00939-0.04405}^{+0.03058+0.03245}$ & $-1.04669_{-0.01327-0.04508}^{+0.03195+0.03669}$ & $-1.20660_{-0.00092-0.01332}^{+0.00660+0.00660}$ \\
\hline$\xi$ & $-0.23758_{-0.08064-0.28867}^{+0.23758+0.23758}$ & $-0.20708_{-0.03706-0.38948}^{+0.20708+0.20708}$ & $-0.09160_{-0.03856-0.11641}^{+0.09160+0.09160}$ & $-0.02830_{-0.00377-0.05692}^{+0.02830+0.02830}$ \\
\hline$\Omega_{m 0}$ & $0.29786_{-0.00930-0.01803}^{+0.00969+0.01723}$ & $0.29640_{-0.00723-0.01474}^{+0.00756+0.01418}$ & $0.29454_{-0.00754-0.01481}^{+0.00764+0.01450}$ & $0.27442_{-0.00663-0.01308}^{+0.00637+0.01300}$ \\
\hline$\sigma_{8}$ & $0.81384_{-0.01288-0.02521}^{+0.01299+0.02568}$ & $0.81543_{-0.01290-0.02615}^{+0.01284+0.02542}$ & $0.81797_{-0.01357-0.02544}^{+0.01409+0.02489}$ & $0.84356_{-0.01284-0.02679}^{+0.01297+0.02624}$ \\
\hline$H_{0}$ & $68.54857_{-0.80198-1.43037}^{+0.76687+1.50829}$ & $68.72657_{-0.72678-1.27043}^{+0.58200+1.35008}$ & $69.04942_{-0.76261-1.41469}^{+0.66552+1.40722}$ & $72.13901_{-0.60082-1.08626}^{+0.56189+1.11083}$ \\
\hline
\end{tabular}

(d) We consider a super phantom dark-energy equation of state as $w_{x} \in[-2,-1.2]$.

For four different priors on the dark-energy equation of state, we present the astronomical constraints on the cosmological parameters in Table IV in which the mean values of the parameters are reported. The two-dimensional contour plots of the parameters $\left(w_{x}, H_{0}\right)$ have been shown in Fig. 13 for four different priors of the dark-energy equation of state. The analyses show that for $w_{x} \in[-1.5,-0.9]$ and $w_{x} \in$ $[-2,-1]$, and $w_{x} \in[-2,-1.01]$, the Hubble constant value is close to the measured value of $H_{0}$ $\left(=67.27 \pm 0.66 \mathrm{~km} \mathrm{~s}^{-1} \mathrm{Mpc}^{-1}\right)$ from Planck [1] and the tension is released at $<2 \sigma$ C.L. On the other hand, for $w_{x} \in[-2,-1.2]$, the result is slightly different because here $H_{0}$ catches the local Hubble constant value measured by Riess et al. $\left(H_{0}=73.24 \pm 1.74 \mathrm{~km} \mathrm{~s}^{-1} \mathrm{Mpc}^{-1}\right)$ [61] within the confidence level slightly greater than $2 \sigma$. So, the tension is not released here. Thus, we see that for the current interaction model the tension can be released. However, one can note that if the dark energy has a strong phantom nature, for instance, here, $w_{x}<-1.2$, then some discrepancies may appear. In summary, the phantom dark-energy equation of state might resolve the current tension on $H_{0}$ in agreement with a recent work [77]; however, the physics with the high phantom dark-energy equation of state
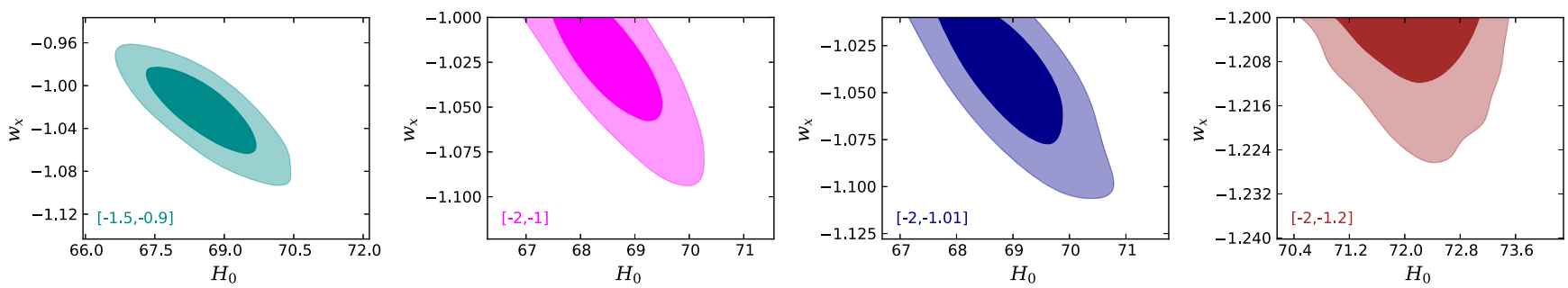

FIG. 13. Contour plots for the parameters $\left(w_{x}, H_{0}\right)$ considering four different regions of the constant dark-energy equation of state. The analysis adopted here is the joint analysis from Planck TT, TE, EE + lowTEB $+\mathrm{BAO}+\mathrm{JLA}+\mathrm{RSD}+\mathrm{WL}+\mathrm{CC}+\mathrm{R} 16$. 
TABLE V. For the dynamical dark-energy equation of state, $w_{x}=w_{0}+w_{a}(1-a)$, we present the results of the interaction model for four different regions of $w_{0}$, the current value of $w_{x}$. The analysis has been performed for the combined analysis Planck TT, TE, $\mathrm{EE}+$ lowTEB $+\mathrm{BAO}+\mathrm{JLA}+\mathrm{RSD}+\mathrm{WL}+\mathrm{CC}+\mathrm{R} 16$. As mentioned earlier, $\Omega_{m 0}=\Omega_{c 0}+\Omega_{b 0}$.

\begin{tabular}{|c|c|c|c|c|}
\hline Parameters & $w_{0} \in[-1.5,-0.9]$ & $w_{0} \in[-2,-1]$ & $w_{0} \in[-2,-1.01]$ & $w_{0} \in[-2,-1.2]$ \\
\hline$\Omega_{c} h^{2}$ & $0.11670_{-0.00120-0.00583}^{+0.00279+0.00422}$ & $0.11630_{-0.00153-0.00484}^{+0.00266+0.00423}$ & $0.11680_{-0.00126-0.00285}^{+0.00159+0.00289}$ & $0.11614_{-0.00266-0.00535}^{+0.00271+0.00528}$ \\
\hline$\Omega_{b} h^{2}$ & $0.02228_{-0.00015-0.00031}^{+0.00015+0.00030}$ & $0.02229_{-0.00014-0.00029}^{+0.00015+0.00029}$ & $0.02229_{-0.00014-0.00028}^{+0.00015+0.00029}$ & $0.02225_{-0.00017-0.00035}^{+0.00018+0.00033}$ \\
\hline $100 \theta_{M C}$ & $1.04069_{-0.00036-0.00064}^{+0.00032+0.00071}$ & $1.04072_{-0.00033-0.00061}^{+0.00032+0.00064}$ & $1.04070_{-0.00030-0.00060}^{+0.00032+0.00060}$ & $1.04071_{-0.00038-0.00072}^{+0.00038+0.00074}$ \\
\hline$\tau$ & $0.06519_{-0.01686-0.03507}^{+0.01664+0.03306}$ & $0.06610_{-0.01802-0.03025}^{+0.01609+0.03227}$ & $0.0666_{-0.01650-0.03087}^{+0.01578+0.03067}$ & $0.06021_{-0.01744-0.03822}^{+0.02037+0.03488}$ \\
\hline$n_{s}$ & $0.97556_{-0.00439-0.00850}^{+0.00448+0.00869}$ & $0.97578_{-0.00428-0.00825}^{+0.00421+0.00802}$ & $0.97613_{-0.00414-0.00737}^{+0.00399+0.00777}$ & $0.97483_{-0.00471-0.01079}^{+0.00577+0.00938}$ \\
\hline $\ln \left(10^{10} A_{s}\right)$ & $3.07082_{-0.03246-0.06887}^{+0.03230+0.06407}$ & $3.07179_{-0.03482-0.05927}^{+0.03156+0.06314}$ & $3.07275_{-0.03112-0.05991}^{+0.03083+0.05896}$ & $3.06041_{-0.03212-0.07292}^{+0.03982+0.06591}$ \\
\hline$w_{0}$ & $-1.02042_{-0.05121-0.14529}^{+0.10065+0.12042}$ & $-1.07026_{-0.01821-0.10996}^{+0.07026+0.07026}$ & $-1.05620_{-0.01190-0.05875}^{+0.04620+0.04620}$ & $-1.22159_{-0.00307-0.04641}^{+0.02159+0.02159}$ \\
\hline$w_{a}$ & $-0.04104_{-0.30556-0.46861}^{+0.22222+0.54574}$ & $0.10561_{-0.20733-0.34558}^{+0.18514+0.38729}$ & $0.07982_{-0.11395-0.25762}^{+0.12230+0.24061}$ & $0.47808_{-0.10169-0.38255}^{+0.19475+0.29336}$ \\
\hline$\xi$ & $-0.27803_{-0.07809-0.50761}^{+0.27803+0.27803}$ & $-0.19671_{-0.03874-0.33102}^{+0.19671+0.19671}$ & $-0.13835_{-0.05235-0.16374}^{+0.13256+0.13835}$ & $-0.09762_{-0.02265-0.15496}^{+0.09762+0.09762}$ \\
\hline$\Omega_{m 0}$ & $0.29657_{-0.00837-0.02268}^{+0.01197+0.02164}$ & $0.29318_{-0.00926-0.01932}^{+0.01031+0.01949}$ & $0.29538_{-0.00807-0.01591}^{+0.00877+0.01602}$ & $0.28267_{-0.00818-0.01598}^{+0.00880+0.01552}$ \\
\hline$\sigma_{8}$ & $0.81234_{-0.01528-0.03984}^{+0.02206+0.04015}$ & $0.81058_{-0.01586-0.03690}^{+0.02005+0.03590}$ & $0.81333_{-0.01234-0.02853}^{+0.01479+0.02705}$ & $0.81078_{-0.02158-0.04294}^{+0.02225+0.04082}$ \\
\hline$H_{0}$ & $68.63729_{-0.88622-1.46090}^{+0.73591+1.61172}$ & $68.93131_{-0.75984-1.54095}^{+0.77984+1.47811}$ & $68.79597_{-0.68156-1.41596}^{+0.71402+1.48429}$ & $\begin{array}{r}70.14999_{-1.06080-1.70609}^{+0.73942+1.95652} \\
\end{array}$ \\
\hline
\end{tabular}

needs further investigation. Finally, we remark that we are dealing with phenomenological models of interaction.

(ii) Dynamical equation of state in $\mathrm{DE}$ and $\mathrm{H}_{0}$ tension: For dynamical dark energy, we perform the same analysis considering four different ranges of $w_{0}$, the current value of the dark-energy equation of state $w_{x}=w_{0}+w_{a}(1-a)$. The constraints on the model parameters have been summarized in Table $\mathrm{V}$, in which their mean values have been shown, while the contour plots for the parameters $\left(w_{0}, H_{0}\right)$ for four different priors on $w_{0}$ have been shown in Fig. 14. We find that the interaction model with dynamical DE preserves behavior almost similar to that of the interaction model with the constant darkenergy equation of state. For $w_{0} \in[-1.5,-0.9]$, $w_{0} \in[-2,-1]$, and $w_{0} \in[-2,-1.01]$, the tension on $H_{0}$ is released within the confidence-level interval $(1 \sigma, 2 \sigma)$. On the other hand, for $w_{0} \in[-2,-1.2]$, the tension is not released. That means IDE 2 has similarities with IDE 1, as we can see.

\section{SUMMARY AND CONCLUSIONS}

The stability analysis of interacting dark-energy models generally divides the parameters space into two separate regions, (i) $w_{x} \geq-1$ and $\xi \geq 0$ or (ii) $w_{x} \leq-1$ and $\xi \leq 0$, in which $w_{x}$ is the state parameter for dark energy and $\xi$ is the coupling parameter of the interaction. That means a discontinuity in the parameters space. A very recent study [71] shows that such difficulties concerning the discontinuity in the parameters space can be solved with a new interaction that includes $\left(1+w_{x}\right)$ in the interaction function $Q$. Such a construction of the interaction function is motivated from the pressure perturbations for dark energy, which informs that the inclusion of such a factor releases the prior on the darkenergy equation of state. That means the factor " $\left(1+w_{x}\right)$ " seems to play a vital role in the analysis of the interacting dark-energy models at the large scale of the universe. Motivated by this fact, in this work, we introduce an interaction, $Q=\xi \dot{\rho}_{x}$, which automatically contains a term $\left(1+w_{x}\right)$ [see Eq. (6)], and consequently, this model holds the same features as in the models of Ref. [71].
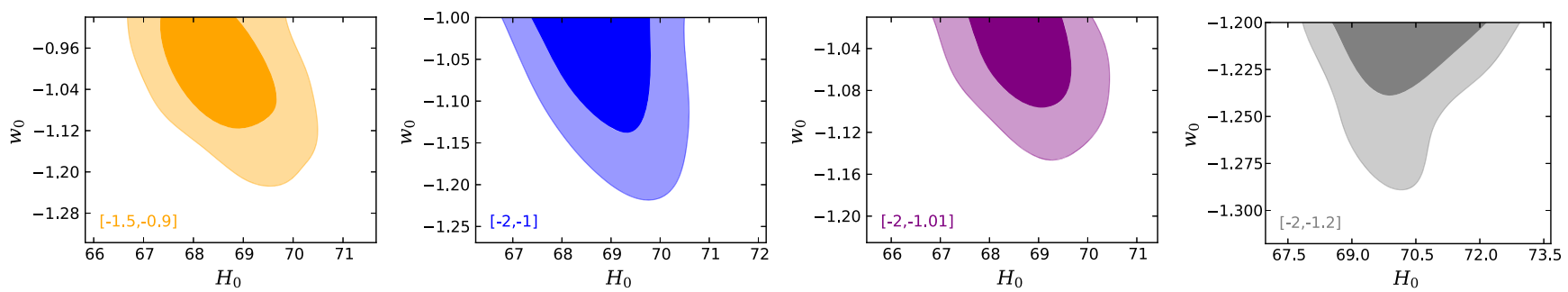

FIG. 14. Contour plots for the parameters $\left(w_{0}, H_{0}\right)$ considering four different regions of $w_{0}$, the current value of the dynamical darkenergy equation of state. The analysis adopted here is the joint analysis from Planck TT, TE, EE + lowTEB $+\mathrm{BAO}+\mathrm{JLA}+$ $\mathrm{RSD}+\mathrm{WL}+\mathrm{CC}+\mathrm{R} 16$. 
We constrained such a typical interaction using the combined astronomical data Planck TT, TE, EE + lowTEB + $\mathrm{BAO}+\mathrm{JLA}+\mathrm{RSD}+\mathrm{WL}+\mathrm{CC}+\mathrm{R} 16$. From Tables I and II, we see that both IDE 1 and IDE 2 allow a very small but nonzero interaction in the dark sectors; however, the zero value of the coupling parameter $\xi$ is not rejected at all. In fact, within $1 \sigma$ confidence level, $\xi=0$ is consistent with the observational data. Further, the analysis also shows that the current value of the darkenergy equation of state (both the mean and the best fit) in both interacting models favors its phantom behavior, while in the $1 \sigma$ confidence level, $w_{x}=-1$, is compatible with the observational data we employ. That means both the interacting models exhibit a very slight deviation from the $\Lambda$ cosmology while within $1 \sigma$ confidence-region $\Lambda$ cosmology is consistent with the observational data. It is also interesting to note that at the perturbative level the models are found to be very close to each other and also to the $\Lambda$ cosmology.

Our analysis also shows that for both IDE 1 and IDE 2 if the Hubble parameter values decrease the dark-energy equation of state shifts its behavior from phantom to quintessence. This behavior was exactly observed in a recent work [71]. It is also interesting to remark that the current IDE models can alleviate the tension on $H_{0}$ that is observed in its global [1] and local measurements [61]. In fact, the theory of interacting dark energy can be a reasonable direction to talk about the current tension on $H_{0}$. We paid a considerable attention to this issue with different regions of the dark-energy equation of state, which concludes that the tension on $H_{0}$ is reconciled in the phantom region, while the analysis also makes a note that the dark-energy equation of state with strong phantom nature needs further attention to reach a definite conclusion.

\section{ACKNOWLEDGMENTS}

The authors thank the referee for some important comments. W. Yang's work is supported by the National Natural Science Foundation of China under Grants No. 11705079 and No. 11647153. The work of S.P. was supported by the SERB-NPDF program (No. PDF/ 2015/000640). D. F. M. acknowledges the support from the Research Council of Norway.
[1] P. A. R. Ade et al. (Planck Collaboration), Astron. Astrophys. 594, A13 (2016).

[2] C. Wetterich, Astron. Astrophys. 301, 321 (1995).

[3] L. Amendola, Phys. Rev. D 62, 043511 (2000).

[4] I. Zlatev, L. M. Wang, and P. J. Steinhardt, Phys. Rev. Lett. 82, 896 (1999).

[5] A. P. Billyard and A. A. Coley, Phys. Rev. D 61, 083503 (2000).

[6] G. Olivares, F. Atrio-Barandela, and D. Pavón, Phys. Rev. D 71, 063523 (2005).

[7] S. del Campo, R. Herrera, and D. Pavón, J. Cosmol. Astropart. Phys. 01 (2009) 020.

[8] L. Amendola et al., arXiv:1606.00180.

[9] T. Koivisto and D. F. Mota, Phys. Lett. B 644, 104 (2007).

[10] S. del Campo, R. Herrera, and D. Pavón, Phys. Rev. D 78, 021302 (2008).

[11] L. P. Chimento, Phys. Rev. D 81, 043525 (2010).

[12] M. Quartin, M. O. Calvao, S. E. Joras, R. R. R. Reis, and I. Waga, J. Cosmol. Astropart. Phys. 05 (2008) 007.

[13] J. Valiviita, R. Maartens, and E. Majerotto, Mon. Not. R. Astron. Soc. 402, 2355 (2010).

[14] T. Clemson, K. Koyama, G. B. Zhao, R. Maartens, and J. Valiviita, Phys. Rev. D 85, 043007 (2012).

[15] S. Pan and S. Chakraborty, Eur. Phys. J. C 73, 2575 (2013).

[16] W. Yang and L. Xu, Phys. Rev. D 90, 083532 (2014).

[17] V. Faraoni, J. B. Dent, and E. N. Saridakis, Phys. Rev. D 90, 063510 (2014).

[18] W. Yang and L. Xu, Phys. Rev. D 89, 083517 (2014).

[19] R. C. Nunes and E. M. Barboza, Gen. Relativ. Gravit. 46, 1820 (2014).
[20] W. Yang and L. Xu, J. Cosmol. Astropart. Phys. 08 (2014) 034.

[21] M. Thorsrud, D. F. Mota, and S. Hervik, J. High Energy Phys. 10 (2012) 066.

[22] J. D. Barrow and D. F. Mota, Classical Quantum Gravity 20, 2045 (2003).

[23] L. Amendola et al., arXiv:1606.00180.

[24] C. Llinares and D. Mota, Phys. Rev. Lett. 110, 161101 (2013).

[25] S. Pan and S. Chakraborty, Int. J. Mod. Phys. D 23, 1450092 (2014).

[26] X. m. Chen, Y. Gong, E. N. Saridakis, and Y. Gong, Int. J. Theor. Phys. 53, 469 (2014).

[27] N. Tamanini, Phys. Rev. D 92, 043524 (2015).

[28] S. Pan, S. Bhattacharya, and S. Chakraborty, Mon. Not. R. Astron. Soc. 452, 3038 (2015).

[29] D. G. A. Duniya, D. Bertacca, and R. Maartens, Phys. Rev. D 91, 063530 (2015).

[30] J. Valiviita and E. Palmgren, J. Cosmol. Astropart. Phys. 07 (2015) 015.

[31] W. Yang, H. Li, Y. Wu, and J. Lu, J. Cosmol. Astropart. Phys. 10 (2016) 007.

[32] S. Pan and G. S. Sharov, Mon. Not. R. Astron. Soc. 472, 4736 (2017).

[33] A. Mukherjee and N. Banerjee, Classical Quantum Gravity 34, 035016 (2017).

[34] J. Solà, J. de Cruz Pérez, A. Gomez-Valent, and R. C. Nunes, arXiv:1606.00450.

[35] G. S. Sharov, S. Bhattacharya, S. Pan, R. C. Nunes, and S. Chakraborty, Mon. Not. R. Astron. Soc. 466, 3497 (2017). 
[36] R. G. Cai, N. Tamanini, and T. Yang, J. Cosmol. Astropart. Phys. 05 (2017) 031.

[37] L. Santos, W. Zhao, E. G. M. Ferreira, and J. Quintin, arXiv: 1707.06827.

[38] J. Mifsud and C. van de Bruck, arXiv:1707.07667.

[39] V. Salvatelli, N. Said, M. Bruni, A. Melchiorri, and D. Wands, Phys. Rev. Lett. 113, 181301 (2014).

[40] R. C. Nunes, S. Pan, and E. N. Saridakis, Phys. Rev. D 94, 023508 (2016).

[41] S. Kumar and R. C. Nunes, Phys. Rev. D 94, 123511 (2016).

[42] C. van de Bruck, J. Mifsud, and J. Morrice, Phys. Rev. D 95, 043513 (2017).

[43] W. Yang, N. Banerjee, and S. Pan, Phys. Rev. D 95, 123527 (2017).

[44] S. Kumar and R. C. Nunes, arXiv:1702.02143.

[45] J. D. Barrow and T. Clifton, Phys. Rev. D 73, 103520 (2006).

[46] R. Adam et al. (Planck Collaboration), Astron. Astrophys. 594, A1 (2016).

[47] N. Aghanim et al. (Planck Collaboration), Astron. Astrophys. 594, A11 (2016).

[48] F. Beutler, C. Blake, M. Colless, D. Heath Jones, L. Staveley-Smith, L. Campbell, Q. Parker, W. Saunders, and F. Watson, Mon. Not. R. Astron. Soc. 416, 3017 (2011).

[49] N. Padmanabhan, X. Xu, D. J. Eisenstein, R. Scalzo, A. J. Cuesta, K. T. Mehta, and E. Kazin, Mon. Not. R. Astron. Soc. 427, 2132 (2012).

[50] M. Manera et al., Mon. Not. R. Astron. Soc. 428, 1036 (2013).

[51] M. Betoule et al. (SDSS Collaboration), Astron. Astrophys. 568, A22 (2014).

[52] C. Heymans et al., Mon. Not. R. Astron. Soc. 432, 2433 (2013).

[53] M. Asgari, C. Heymans, C. Blake, J. Harnois-Deraps, P. Schneider, and L. Van Waerbeke, Mon. Not. R. Astron. Soc. 464, 1676 (2017).

[54] W. J. Percival et al. (2dFGRS Collaboration), Mon. Not. R. Astron. Soc. 353, 1201 (2004).
[55] C. Blake et al., Mon. Not. R. Astron. Soc. 415, 2876 (2011).

[56] L. Samushia, W. J. Percival, and A. Raccanelli, Mon. Not. R. Astron. Soc. 420, 2102 (2012).

[57] B. A. Reid et al., Mon. Not. R. Astron. Soc. 426, 2719 (2012).

[58] F. Beutler, C. Blake, M. Colless, D. Heath Jones, L. Staveley-Smith, G. B. Poole, L. Campbell, Q. Parker, W. Saunders, and F. Watson, Mon. Not. R. Astron. Soc. 423, 3430 (2012).

[59] S. de la Torre et al., Astron. Astrophys. 557, A54 (2013).

[60] M. Moresco, L. Pozzetti, A. Cimatti, R. Jimenez, C. Maraston, L. Verde, D. Thomas, A. Citro, R. Tojeiro, and D. Wilkinson, J. Cosmol. Astropart. Phys. 05 (2016) 014.

[61] A. G. Riess et al., Astrophys. J. 826, 56 (2016).

[62] M. Chevallier and D. Polarski, Int. J. Mod. Phys. D 10, 213 (2001).

[63] E. V. Linder, Phys. Rev. Lett. 90, 091301 (2003).

[64] C. P. Ma and E. Berschinger, Astrophys. J. 455, 7 (1995).

[65] V. F. Mukhanov, H. A. Feldman, and R. H. Brandenberger, Phys. Rep. 215, 203 (1992).

[66] K. A. Malik and D. Wands, Phys. Rep. 475, 1 (2009).

[67] J. Valiviita, E. Majerotto, and R. Maartens, J. Cosmol. Astropart. Phys. 07 (2008) 020.

[68] E. Majerotto, J. Valiviita, and R. Maartens, Mon. Not. R. Astron. Soc. 402, 2344 (2010).

[69] M. B. Gavela, L. Lopez Honorez, O. Mena, and S. Rigolin, J. Cosmol. Astropart. Phys. 11 (2010) 044.

[70] M. B. Gavela, D. Hernandez, L. Lopez Honorez, O. Mena, and S. Rigolin, J. Cosmol. Astropart. Phys. 07 (2009) 034.

[71] W. Yang, S. Pan, and J. D. Barrow, arXiv:1706.04953.

[72] K. Koyama, R. Maartens, and Y. S. Song, J. Cosmol. Astropart. Phys. 10 (2009) 017.

[73] A. Lewis and S. Bridle, Phys. Rev. D 66, 103511 (2002).

[74] J. D. Barrow, Phys. Rev. D 89, 064022 (2014).

[75] W. Lin and M. Ishak, Phys. Rev. D 96, 023532 (2017).

[76] W. Lin and M. Ishak, Phys. Rev. D 96, 083532 (2017).

[77] E. Di Valentino, A. Melchiorri, and O. Mena, Phys. Rev. D 96, 043503 (2017). 